\title{
Granular avalanches of entangled rigid particles
}

\author{
Damien P. Huet $\odot$ \\ Department of Mathematics, University of British Columbia, 1984 Mathematics Road, \\ Vancouver, British Columbia, Canada V6T 1 Z2 \\ Maziyar Jalaal \\ van der Waals-Zeeman Institute, Institute of Physics, University of Amsterdam, \\ Science Park 904, 1098 XH Amsterdam, Netherlands \\ Rick van Beek @ and Devaraj van der Meer \\ Physics of Fluids Group, Max Planck Center for Complex Fluid Dynamics, \\ MESA+ Institute and J.M. Burgers Centre for Fluid Dynamics, University of Twente, \\ P.O. Box 217, 7500 AE Enschede, Netherlands \\ Anthony Wachs $\oplus^{*}$ \\ Department of Mathematics, University of British Columbia, 1984 Mathematics Road, \\ Vancouver, British Columbia, Canada V6T $1 Z 2$ \\ and Department of Chemical and Biological Engineering, University of British Columbia, 2360 E Mall, \\ Vancouver, British Columbia, Canada V6T 1 Z3
}

(Received 23 February 2021; accepted 21 September 2021; published 8 October 2021)

\begin{abstract}
In granular mechanics, the shape of grains plays a critical role in the overall dynamics and significantly affects the macroscopic properties of the system. Using a dam break setup, granular collapses of nonconvex (cross-shaped) plastic particles assumed quasirigid are conducted experimentally and simulated numerically for a wide range of aspect ratios $a=H_{0} / L_{0}$, with $H_{0}$ the initial height of the column and $L_{0}$ its initial length. We report avalanche dynamics such as the top-driven collapse and the buckling collapse, as well as an intermittent flow behavior where reproducibility is lost and where the stability of the column is determined by the random initial configuration of the assembly of entangled particles. While counterintuitive and despite fundamentally different dynamics, we find that the runout distance $L_{\infty}$ and the final height $H_{\infty}$ of our granular collapses of crosses agree with those of spherical particles both experimentally and numerically. Our discrete element method simulations are able to reproduce all flow behaviors observed experimentally and they show excellent quantitative agreement with the experimental data. In the simulations, extra care is given to adopting a tangential friction force model based on the cumulative tangential displacement at the contact point, critical to represent stable cases, and to determining the contact model parameters. The analysis of (i) the force network via the average probability density function of contact force magnitude and (ii) the fabric anisotropy suggests that the stability of the column is a complex problem determined by mesoscale properties that we could not reliably identify at that point.
\end{abstract}

DOI: 10.1103/PhysRevFluids.6.104304

*wachs@mail.ubc.ca 


\section{INTRODUCTION}

Granular materials are still far from being well understood despite their ubiquitous presence in natural and industrial processes. Yet, in many applications it is critical to have reliable tools to provide both qualitative and quantitative information about granular materials. The information sought can include flow conditions, jamming or buckling effects, and stability of a granular assembly, as well as the flow rate of a hopper discharge, the runout distance of a landslide, or the added mass on walls from the Janssen effect [1]. In the past two decades there has been a growing interest in the study of granular systems of spherical particles. In the case of rectangular and axisymmetric dam break configurations, Lajeunesse et al. [2] have provided experimental results and a force-balance model that correctly reproduces the scaling of the runout distance as a function of the initial aspect ratio. Balmforth and Kerswell [3] also conducted dam break experiments and Kerswell [4] developed a depth-average model to explain their observations. In the meantime, the discrete element method (DEM) gained popularity with the increase of computing power. In most cases, the particle shape is simplified to spheres, ellipsoids, or even composite particles made of overlapping spheres. However, numerous studies [5-7] show that the particle blockiness, particle surface roughness, and particle aspect ratio have a major impact on the dynamics and the packing properties of the considered systems. Nonconvex particles are even less represented in experimental and numerical studies, even though most natural processes involve arbitrary shapes, including nonconvex ones. It is reasonable to expect that the flow properties and steady-state quantities are significantly different in the case of convex and nonconvex particle shapes, as Rémond et al. [8], Gravish et al. [9], and more recently Landauer et al. [10] and Wang et al. [11] have observed with packings of nonconvex particles.

When studying granular materials, dam break collapses on a horizontal flat surface have been popular due to the relative simplicity of this setup and the rich physics that granular media exhibit in this configuration. Staron and Hinch [12] used a two-dimensional discrete element method to study the collapse and spreading of disk-shaped particles onto a horizontal plane. They showed that for initial aspect ratios $a \gtrsim 2.5$, the column has a period of free fall, whereas columns with $a \lesssim 2.5$ do not. This observation explains the existence of two distinct scaling laws for the runout distance versus the initial aspect ratio, as previously reported by Lajeunesse et al. [2] and Lube et al. $[13,14]$. It also highlights that the runout distance not only is dependent on effective flow properties such as basal friction, but is also strongly affected by the fall dynamics. Moving to three-dimensional simulations, Lacaze et al. [15] proposed a comparison between DEM simulations and experimental results in the case of a planar channel, of width less than two particle diameters. The response they obtained exhibits a flowing top layer and their DEM simulations are in good qualitative and quantitative agreement with their experiments. Furthermore, they already highlighted the difficulty of assigning values to friction parameters in their DEM simulations and the need to rely on calibration experiments. Girolami et al. [16] later performed fully three-dimensional avalanches of spherical particles with DEM simulations and compared their numerical results to the experiments of Lajeunesse et al. [2]. The quantitative scaling with the initial height-to-width aspect ratio and the qualitative flow structure and scaled free surface show very good agreement with the experimental data of Lajeunesse et al. Girolami et al. [17] later used DEM simulations to study unchannelized dam break flows and showed that the runout distance versus initial aspect ratio scaling power lies in between that of previously reported channelized and axisymmetric flows. With regard to numerical simulations of avalanches of nonspherical particles, Lim et al. [18] studied two-dimensional collapses of spheres, convex particles, and nonconvex gritlike particles. Although not compared to experimental data, their results revealed that the runout distance and angle of repose are greater for nonconvex particles than for convex particles and spheres. Finally, Rakotonirina and Wachs [19] considered granular slumpings of a realistic number of particles, over $2 \times 10^{6}$, in channels of periodic and finite width. Claiming their icosahedral particles have similar shapes to those used in the experiments of Lube et al. [14] and Balmforth and Kerswell [3], they quantitatively compared the runout distance and its scaling law versus the initial aspect ratio to those experiments. 
They obtained very good agreement with the data reported by Balmforth and Kerswell and an overestimated runout distance when compared to the experimental results of Lube et al., highlighting the relatively wide range of reported data.

On the computational side, the past decade has seen rapid developments in the field of DEM of nonspherical particles, and we will recall briefly here the main methods and recent contributions relevant to the simulations of nonspherical particles. When the shape of grains deviates from disks or spheres, the first challenge is to describe the shape. While it is possible to mesh the surface of each particle, the computational cost of the contact detection algorithm limits this approach to a small number of particles [20,21], and other approaches are preferred for large granular systems. Ellipses and superquadric shapes were among the first nonspherical shapes to be considered in DEM simulations [22-24]. Superquadric shapes still receive considerable interest as they offer a unified framework to represent a wide range of shapes [25-27]. However, with greater blockiness the contact detection algorithm of superquadric shapes become more and more costly. As a result, in the case of particles that have faces and edges, a polyhedral representation as in $[19,28-30]$ is better suited than high-order, i.e., "sharp," superquadrics. In terms of physics, we note that most of the studies regarding nonspherical particles, and especially regarding nonconvex particles, focus on random packings (see, e.g., [8-11,30,31]), resulting in a gap between our understanding of static and dynamic assemblies of nonspherical particles. With regard to static assemblies of nonspherical particles, Seelen et al. [30] presented a unified framework to treat any convex shape including cuboids, ellipsoids, and more complex polyhedra that are close to sand grains and woodchips. They investigated the packing fraction as a function of the distance to the wall and reported a strong influence of the aspect ratio of ellipsoids and cylinders. Packings of nonconvex particles have been studied by Ludewig and Vandewalle [31], Gravish et al. [9], Rakotonirina et al. [29], and Landauer et al. [10], among others. Ludewig and Vandewalle considered the compaction of nonconvex particles made of composite overlapping spheres with varying sphericity. They showed that decreasing the sphericity of the shapes, which in the case of their study results in increasing their concavity, leads to more interlocking of the granular assembly, measured in terms of the mobility of the particles. Furthermore, they showed that the greater the concavity of the grains is, the greater the stability of the whole assembly is despite a lower packing fraction. More recently, Landauer et al. [10] analyzed the bulk and tapping densities as well as the angle of repose of granular assemblies of various shapes, including the nonconvex stellated octahedron, tetrapod, and quadropod shapes. Their work belongs to the few DEM studies $[9,29,32,33]$ that validate their simulations of nonconvex particles with experiments, and as such they highlight the difficulty to find contact parameters and conclude that calibration is necessary for every shape. We face here the same difficulty with assigning values to our friction model parameters. We discuss this issue in Sec. V C. On the dynamic behavior of nonspherical particles, Rakotonirina et al. [29] considered rotating drums filled with particles of spherical, convex and nonconvex shapes and reported a wide range of flow regimes depending on the angular velocity of the rotating drum, the nonconvex shape exhibiting the greatest complexity and variety of regimes. Govender et al. [33] showed with experiments of three-dimensionally printed nonconvex particles and GPU-based DEM simulations that in the case of a hopper discharge the dynamic behavior of the assembly is strongly impacted by the concavity of the grains, since the nonconvexity gives rise to jamming effects. Interestingly, the authors of [33] were able to observe significant differences in the flow behavior with Schönhardt polyhedra compared to triangular prisms, while a Schönhardt polyhedron only slightly differs from its convex counterpart, the triangular prism. However, due to hardware limitations, the force model used in their studies does not include a tangential memory friction term, which, we will see in Sec. VB, is crucial to reproduce static states.

In this study we investigate experimentally and numerically the behavior of granular materials beyond the well-known spherical or convex shapes, as a step forward in the pursuit of modeling realistic granular media. We choose nonconvex particles with a large aspect ratio that are very prone to entanglement and we perform dam break experiments and simulations. We report flow behaviors typical of particle entanglement. Our traditional CPU-based architecture allows us to 
include a memory friction term in order to accurately reproduce stable configurations of particles of any shape, including of nonconvex shape. We show that for the sole dam break setup, considering nonconvex particles results in a wide range of granular behaviors that are very different than those observed from avalanches of convex particles, such as the top-driven collapse or the intermittent regime, a regime where reproducibility is lost and where the flow outcome is determined by the random initial particle configuration. We perform DEM simulations of our experimental setup using the code GRAINS3D [19,28,29] and we show that our numerical simulations accurately reproduce both qualitative and quantitative results. Then we explore the information provided by the microstructure and the force network, we highlight the importance of the memory term in the tangential friction model, and we discuss the choice of contact parameters.

The rest of the paper is organized as follows. Section II gives an overview of our own DEM version including contact detection for nonspherical rigid bodies, contact force model, and contact parameter determination. In Sec. III we present the flow configuration and describe the experimental equipment used to record the granular collapses. We analyze experimental and numerical results in Sec. IV in terms of dynamics of the collapse, flow map, final height, runout distance, time evolution, force network, and fabric anisotropy. We then discuss in Sec. V the key elements of the DEM that allow for a quantitative match of the numerical results with the experimental data. We summarize in Sec. VI.

\section{NUMERICAL METHOD}

\section{A. General structure of the discrete element method}

The numerical simulations in this study are performed using the standard soft-body DEM as introduced by Cundall and Strack [34]. In the DEM, each particle is tracked individually, and the position and velocity of particle $i$ are determined by explicitly integrating Newton's second law of motion

$$
\begin{aligned}
\frac{d \boldsymbol{v}_{i}}{d t} & =\frac{\boldsymbol{F}_{i, \mathrm{ext}}}{m_{i}}, \\
\frac{d \boldsymbol{x}_{i}}{d t} & =\boldsymbol{v}_{i}, \\
\frac{d \mathcal{J}_{i} \cdot \boldsymbol{\omega}_{i}}{d t} & =\boldsymbol{M}_{i, \mathrm{ext}}, \\
\frac{d \boldsymbol{\theta}_{i}}{d t} & =\boldsymbol{\omega}_{i},
\end{aligned}
$$

where $t$ is the time, $\boldsymbol{x}_{i}$ is the position of the center of mass of particle $i, m_{i}$ is its mass, $\boldsymbol{v}_{i}$ is its velocity, $\mathcal{J}_{i}$ is its inertia tensor, $\boldsymbol{\theta}_{i}$ is its angular position, $\boldsymbol{\omega}_{i}$ is its angular velocity, and $\boldsymbol{F}_{i, \mathrm{ext}}$ and $\boldsymbol{M}_{i, \text { ext }}$ are the sums of external forces and torques exerted on the particle, respectively.

A change of momentum for a given particle is due to the action of body forces, such as gravity, as well as interparticle or particle-wall contact forces. Figure 1 shows a top-level description of a typical DEM algorithm that can be summarized in three steps to be performed at each time step and for each particle: (i) the contact detection, (ii) the computation of forces due to interparticle or particle-wall contacts, and (iii) the numerical integration of the equations of motion (1)-(4).

Step (i) is a purely computational problem and its complexity depends on the shape of the particles at play. In the case of spherical particles, the contact is trivially assessed from the position of the centers of mass of the two potentially contacting spheres, leading to a very fast detection (the same applies to a sphere-infinite plane contact). Several approaches are possible in order to handle the detection of contacts between nonspherical particles. The method of glued spheres involves approaching any shape by a cluster of $N$ overlapping spheres. Two glued-spheres particles are in contact if at least two of their subspheres overlap. As $N$ increases, the approximation of the shape improves but the contact detection becomes more computationally expensive as it scales 


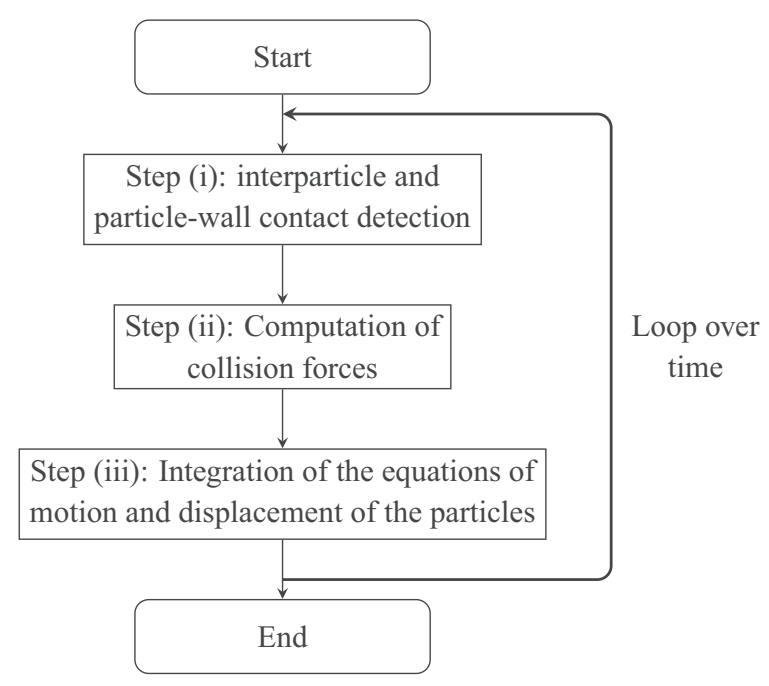

FIG. 1. Top-level view of a soft-body DEM algorithm.

with $N^{2}$. Several studies $[29,35,36]$ have mentioned that the dynamics is severely perturbed by the artificial surface roughness, and Ref. [35] shows that having $N$ of the order of $10^{2}$ is still not enough to obtain realistic trajectories for a single quasisphere bouncing on a flat surface. This makes the method of glued spheres out of reach, or at least extremely inefficient with respect to the following methods. If particles are convex polyhedra, the common plane algorithm as used by Cundall [37] and later improved by Nezami et al. [38] has shown a remarkable performance and stability. At the price of rounded edges, particles of arbitrary shapes can also be described by superquadric functions. In that case, the contact detection between two superquadric shapes is an optimization problem, the complexity of which depends on the level of angularity of the shapes [27]. For applications where angular and nonpolytope particles need to be described, the most versatile contact detection algorithm has been proposed by Gilbert et al. [39]. It involves solving iteratively small linear systems of size 4 (in three dimensions) until a reasonable approximation of the contact point has been found. Our DEM solver GRAINS3D uses the Van den Bergen implementation of this so-called Gilbert-Johnson-Keerthi (GJK) algorithm [40]. Since the GJK algorithm is limited to convex bodies, nonconvex particles are handled by decomposing their shape into a set of elementary convex particles on which the GJK algorithm can be performed successively, as described in [29].

Step (ii) will be discussed in detail in the next section. For an extensive discussion of step (iii), the interested reader is referred to the review of Džiugys and Peters [41] and the work of KruggelEmden et al. [35]; for a short summary of the various integrators used in the DEM one can refer to Sec. 3.3.2 in Ref. [42]. In this study, the classical second-order velocity Verlet integrator is adopted.

\section{B. Contact model}

In the soft-body DEM, the computation of contact forces is critical as it determines the mechanical response of the system. Contact forces are computed using geometrical features of the contact region, defined in Fig. 2. As shown in Fig. 2, the contact is modeled as an overlap of the particle shapes. We denote by $\boldsymbol{u}_{\boldsymbol{n}}$ and $\boldsymbol{u}_{\boldsymbol{t}}$ the unitary normal and tangential vectors, respectively, at the contact point, by $C$ the point of application of the force, and by $\delta_{n}$ the penetration depth, also called overlapping distance. Note that the point $C$ here is chosen as the midpoint of the segment $\overline{A B}$ formed by the two points that define the greatest penetration depth $\delta_{n}$. While this choice is natural for spherical particles, the point of application of the force is less straightforward for particles of complex shape, especially when the physical force is not applied at a single point but is distributed 


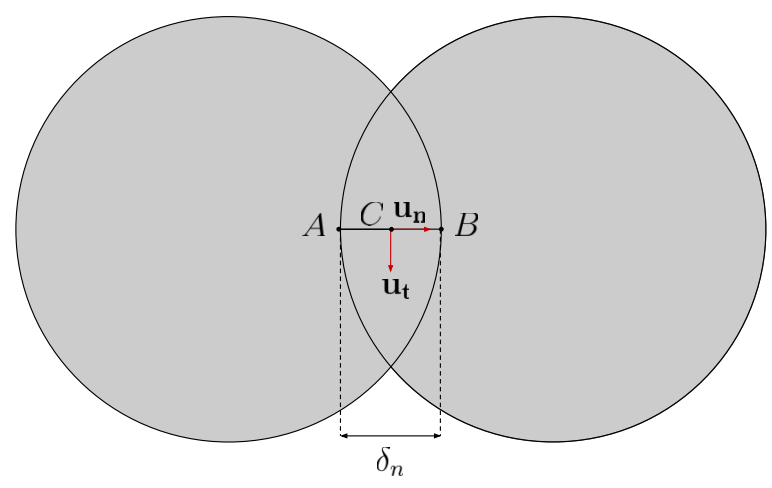

FIG. 2. In soft-body DEM, a contact is modeled with an overlap (note that the overlap is intentionally magnified here for visual purposes).

over a line or a surface. Seelen et al. [30] discussed ways to impose a physically meaningful contact point for most line- and surface-distributed force configurations. In this study, we consider the occurrence of face-face and face-edge contacts to be minimal with respect to the rest of the contact configurations and model face-face and face-edge contacts with a single contact point similarly to the way we model the other contact configurations. Discrete element methods that represent particles as triangulated surfaces [20,21] or by a discrete function representation [24,26] are naturally able to distribute the force over the multiple contact points of one particle that by construction coincide with nodes of a triangulated face that belong to the other particle and vice versa.

Once the point of application of the contact force is defined, two contact models are typically used to compute the contact forces: the Hertz dashpot model and the linear spring-dashpot model. While the former has the advantage of being analytically derived for the contact of two spheres and other simple shapes, such as a sphere-plane configuration, there is no analytical generalization to arbitrary shapes, therefore excluding its use in this study. As a result, we adopt the latter springdashpot model (its schematic view is presented in Fig. 3) and we elaborate below on its formulation and implementation in translation both in the normal direction and in the tangential direction. The formulation of the contact model in rotation is given in Appendix A 1.

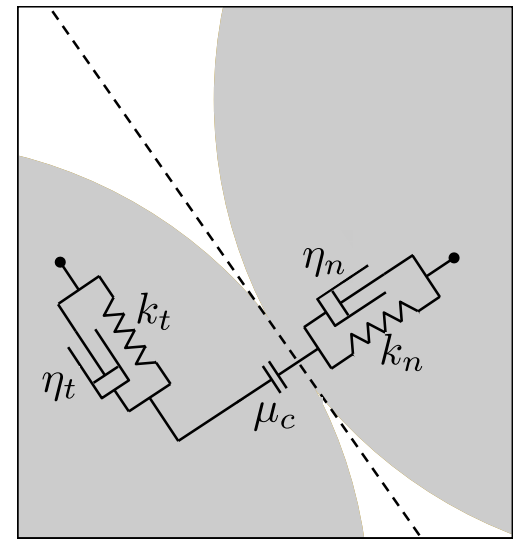

(a)

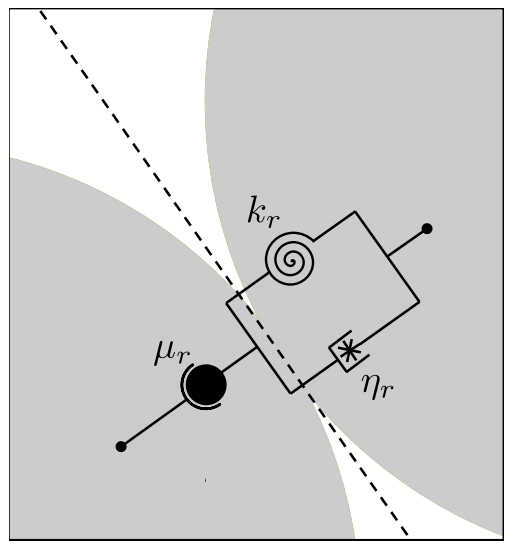

(b)

FIG. 3. Linear spring-dashpot contact model as a mechanical analog in (a) translation and (b) rotation. The dashed line represents the tangential contact plane. 


\section{Contact model in translation}

The normal contact force $\boldsymbol{F}_{\boldsymbol{n}}$ is expressed as

$$
\boldsymbol{F}_{\boldsymbol{n}}=\max \left(k_{n} \delta_{n}+\eta_{n} \frac{d \delta_{n}}{d t}, 0\right) \boldsymbol{u}_{\boldsymbol{n}}
$$

where $k_{n}$ and $\eta_{n}$ are the normal stiffness coefficient and the normal damping coefficient, respectively. The normal force represents the viscoelastic response to the contact and is intrinsically repulsive. Note that at the end of the contact, the term $k_{n} \delta_{n}$ could become smaller in absolute value than the negative term $\eta_{n} d \delta_{n} / d t$. Therefore, the role of the $\max (\cdot, 0)$ condition is to prevent $\boldsymbol{F}_{\boldsymbol{n}}$ from becoming an attractive force.

The tangential friction force $\boldsymbol{F}_{\boldsymbol{t}}$ is also modeled as a linear spring dashpot, though slightly modified to allow for the Coulomb friction saturation,

$$
\begin{gathered}
\boldsymbol{F}_{t}=\min \left(\left\|-k_{t} \boldsymbol{\delta}_{t}-\eta_{t} \boldsymbol{v}_{t}\right\|, \mu_{c}\left\|\boldsymbol{F}_{n}\right\|\right) \boldsymbol{u}_{t, \mathrm{cumul}}, \\
\boldsymbol{u}_{t, \mathrm{cumul}}=-\frac{k_{t} \boldsymbol{\delta}_{t}+\eta_{t} \boldsymbol{v}_{t}}{\left\|k_{t} \boldsymbol{\delta}_{t}+\eta_{t} \boldsymbol{v}_{t}\right\|},
\end{gathered}
$$

where $\boldsymbol{u}_{t, \text { cumul }}$ is the cumulative tangential unit vector, $\boldsymbol{v}_{t}$ is the tangential relative velocity, $k_{t}$ and $\eta_{t}$ are the tangential stiffness coefficient and the tangential damping coefficient, respectively, and $\boldsymbol{\delta}_{t}$ is the cumulative tangential displacement. The reason $\boldsymbol{u}_{t, \text { cumul }}$ is introduced instead of simply having $\boldsymbol{F}_{t}$ in the direction of $-\boldsymbol{v}_{t}$ is to keep a meaningful tangential direction even when the system reaches a static state, i.e., when $\left\|\boldsymbol{v}_{t}\right\|$ approaches the machine epsilon. The term $\boldsymbol{\delta}_{t}$ is often referred to as the history or memory term because it stores information about the contact at previous instants. It is expressed as

$$
\boldsymbol{\delta}_{t}= \begin{cases}q_{\mathrm{rot}} \boldsymbol{\delta}_{t}^{t-\Delta t} q_{\mathrm{rot}}^{-1}+\int_{t}^{t+\Delta t} \boldsymbol{v}_{t}(s) d s & \text { if }\left\|F_{t}\right\| \leqslant \mu_{c}\left\|F_{n}\right\| \\ \frac{-\mu_{c}\left\|F_{n}\right\| \boldsymbol{u}_{t, \mathrm{cumul}}-\eta_{t} \boldsymbol{v}_{t}}{k_{t}} & \text { if }\left\|F_{t}\right\|>\mu_{c}\left\|F_{n}\right\| .\end{cases}
$$

In Eq. (8), $\delta_{t}^{t-\Delta t}$ is the cumulative tangential displacement at the previous instant (if the contact existed), $q_{\text {rot }}$ is the rotation quaternion from the tangential plane at the previous instant to the current tangential plane, and $\mu_{c}$ is the Coulomb sliding coefficient. If we neglect the rotation of the tangential plane, $\boldsymbol{\delta}_{t}$ becomes $\int_{t_{0}}^{t} \boldsymbol{v}_{t}(s) d s$ and can be seen as the length of the contact path since the contact start time $t_{0}$. This is at least true until $\left\|F_{t}\right\|$ reaches its saturation value $\mu_{c}\left\|\boldsymbol{F}_{\boldsymbol{n}}\right\|$. If the tangential friction desaturates, starting a new path integral $\int_{0}^{\Delta t} \boldsymbol{v}_{t}(s) d s$ would result in a sudden and unphysical discontinuity in $\boldsymbol{F}_{\boldsymbol{t}}$. Defining $\boldsymbol{\delta}_{t}$ as in Eq. (8) ensures that the tangential friction will remain continuous through desaturation $[43,44]$.

\section{Contact model parameters}

The above contact model requires setting five parameters: the stiffness and damping coefficients $k_{i}$ and $\eta_{i}$ in both translational directions, as well as the static friction coefficient $\mu_{c}$. If a rotational friction is considered, three additional coefficients need to be determined, as introduced in Appendix A 1. Most of these coefficients do not have a clear physical meaning, and assigning meaningful values to them is not a straightforward task. As a result, there is no consensus in the literature on a well-defined and universal methodology to determine the magnitude of contact model parameters, and most of these values have generally been established for spherical particles only. We present below our procedure that is designed by carefully reviewing the literature and assembling parts of previously existing procedures which require the least amount of hypothesis or sloppy formulations. A procedure to set the contact parameters in rotation is given in Appendix A 2. 


\section{Limitations of the intuitive and physical approach}

From Hooke's law and Hertz theory and following Džiugys and Peters [41] [Eq. (43) in their study], one can relate material properties to some contact model parameters such as the normal stiffness $k_{n}$,

$$
k_{n}=\frac{4}{3} \frac{E_{i} E_{j}}{E_{i}\left(1-\sigma_{j}^{2}\right)+E_{j}\left(1-\sigma_{i}^{2}\right)} R_{i j},
$$

or for two particles of the same material

$$
k_{n}=\frac{2 E_{i} R_{i j}}{3\left(1-\sigma^{2}\right)},
$$

where $E_{i}$ and $\sigma_{i}$ are Young's modulus and the Poisson ratio, respectively, of material $i$. Then it is not difficult to find the expression of the normal restitution coefficient $e_{n}$ [28],

$$
e_{n}=\exp \left(-\gamma_{n} \frac{\pi}{\sqrt{\omega_{0}^{2}-\gamma_{n}^{2}}}\right),
$$

where $\gamma_{n}=\eta_{n} / 2 m_{i j}$ and $\omega_{0}^{2}=k_{n} / m_{i j}$ is the resonance frequency of the system, with $m_{i j}=$ $m_{i} m_{j} /\left(m_{i}+m_{j}\right)$ the reduced mass of the two contacting bodies.

The time step $\Delta t$ is set according to the contact time $T_{c}$. As summarized by Kruggel-Emden et al. [35], the number of recommended time steps per contact varies strongly from author to author and is often set regardless of the integration scheme. From the analysis of the accuracy of dozens of integration schemes, Kruggel-Emden et al. [35] found that having 20 time steps per contact is typically sufficient to get a resolved enough simulation. In a simple two-sphere contact case, the contact time is expressed as

$$
T_{c}=\frac{\pi}{\sqrt{\omega_{0}^{2}-\gamma_{n}^{2}}}=\sqrt{\frac{m_{i j}\left[\pi^{2}+\ln \left(e_{n}\right)^{2}\right]}{k_{n}}} .
$$

For steel, typically $E=210 \mathrm{GPa}$ and $\sigma=0.3$. Considering two 1-mm steel balls contacting, we find $k_{n}=6.37 \times 10^{8} \mathrm{~N} / \mathrm{m}$. This means that the contact time $T_{c}$ is close to $5 \times 10^{-7} \mathrm{~s}$, imposing $\Delta t \leqslant 2.5 \times 10^{-8} \mathrm{~s}$. For computational reasons, such a small time step is practically unachievable.

\section{Procedure to find contact parameters in translation}

At this stage, performing reliable DEM simulations can seem out of reach. However, it has been shown by Yan et al. [45] and Cleary [6] that for rigid particles a physical value for the normal stiffness $k_{n}$ does not play a significant role in the overall dynamics of the system and that decreasing its value by orders of magnitudes, depending on the system, can be safely performed. In fact, it is not the actual stiffness that drives the behavior of the granular medium, and decreasing its value primarily increases the contact time $T_{c}$, allowing for larger time steps $\Delta t$. While the artificially lengthened contact time $T_{c}$ is kept much smaller that the timescale of the system, the dynamics of the granular medium is unchanged. For a given force exerted on a particle, the stiffness also controls the extent of overlap between the contacting particles that is of major importance for obvious geometric reasons. On the basis of this approach, contact model parameters are set using the following procedure.

Step 1: Determine values for $\delta_{\max }, e_{n}, e_{t}$, and $\mu_{c}$. Based on empirical observations, Yan et al. [45] and Cleary [6] concluded that an appropriate particle overlap should remain below $0.5 \%$ of the particle radius. The restitution coefficient $e_{n}$ lies by definition in the range $0-1$ and its value is easy to measure experimentally, at least for spheres. The sliding friction coefficient is also straightforward 
to measure experimentally and typically ranges from 0 to 1.5 . The tangential restitution coefficient $e_{t}$ is a less straightforward parameter to measure and can be set with a calibration procedure.

Step 2: Compute $k_{n}$ from an estimated maximum impact velocity $v_{\text {max }}$. Solving analytically for the overlap $\delta_{n}$ in a simple two-sphere gravityless contact case and computing its maximum value $\delta_{\max }$ leads to

$$
\delta_{\max }=\frac{v_{\max }}{\omega} e^{-\gamma_{n} T_{\max }} \sin \left(\omega T_{\max }\right),
$$

with

$$
\omega=\sqrt{\omega_{0}^{2}-\gamma_{n}^{2}}=\sqrt{\frac{k_{n}}{m_{i j}}-\gamma_{n}^{2}}
$$

and

$$
T_{\max }=\frac{1}{\omega} \arctan (\zeta), \quad \zeta=\frac{\omega}{\gamma_{n}}=-\frac{\pi}{\ln \left(e_{n}\right)} .
$$

From Eq. (13) we can solve for $k_{n}$ :

$$
k_{n}=m_{i j}\left(1+\frac{1}{\zeta^{2}}\right)\left(\frac{v_{\max }}{\delta_{\max }} e^{-(1 / \zeta) \arctan (\zeta)} \sin [\arctan (\zeta)]\right)^{2}
$$

Step 3: Compute the contact time $T_{c}$ and the normal damping coefficient. The contact time $T_{c}$ is computed from Eq. (12). Then, using Eq. (11), the normal damping coefficient $\eta_{n}$ is given by

$$
\eta_{n}=-2 m_{i j} \frac{\omega_{0} \ln \left(e_{n}\right)}{\sqrt{\pi^{2}+\left[\ln \left(e_{n}\right)\right]^{2}}}
$$

Step 4: Compute the tangential parameters $k_{t}$ and $\eta_{t}$. Following Pournin et al. [46] [Eq. (22) therein], the tangential model parameters can be linked to the normal parameters as

$$
\begin{gathered}
k_{t}=\frac{k_{n}}{1+m_{i j}\left(\frac{R_{i}^{2}}{I_{i}}+\frac{R_{j}^{2}}{I_{j}}\right)} \frac{\pi^{2}+\ln \left(e_{t}\right)^{2}}{\pi^{2}+\ln \left(e_{n}\right)^{2}}, \\
\eta_{t}=\frac{\eta_{n}}{1+m_{i j}\left(\frac{R_{i}^{2}}{I_{i}}+\frac{R_{j}^{2}}{I_{j}}\right)} \frac{\ln \left(e_{t}\right)}{\ln \left(e_{n}\right)},
\end{gathered}
$$

where $I_{i}$ and $I_{j}$ are the moments of inertia about the rolling axes of the particles.

At this point we remind the reader that the above procedure to find translational parameters has been derived for spherical particles and that an extension to nonspherical particles is not straightforward a priori. In the case of ellipsoids, Seelen et al. [30] modified this expression by replacing the radii $R_{1}$ and $R_{2}$ by the distance between the contact point and the center of gravity of the two ellipsoids. We discuss this extension to nonspherical bodies in Sec. V C.

\section{EXPERIMENTAL AND NUMERICAL SETUP}

\section{A. Experimental setup}

The tests are performed in a dam break setup sketched in Fig. 4(a). The box is made of acrylic and its dimensions are $L \times H \times W=64 \times 30 \times 20 \mathrm{~cm}^{3}$. The setup features a sliding gate (made of PVC). Slots are milled on the sidewalls to guide the vertical motion of the gate and therefore its location can be easily adjusted to $L=4,8,12,20$, and $30 \mathrm{~cm}$, or $L \approx 1.5 L_{p}, 3.0 L_{p}, 4.4 L_{p}$, $7.4 L_{p}$, and $11.1 L_{p}$ respectively, where $L_{p}=27 \mathrm{~mm}$ is the characteristic length of the particles shown in Fig. 4(b). A pneumatic cylinder is used to control the release of the gate. The gate releases with an (almost constant) acceleration of $43 \pm 3 \mathrm{~m} / \mathrm{s}^{2}$, reaching the speed of $\sim 3 \pm 0.1 \mathrm{~m} / \mathrm{s}$ in $0.07 \mathrm{~s}$ (calculated by image processing). The frames holding the pneumatic cylinder are placed 


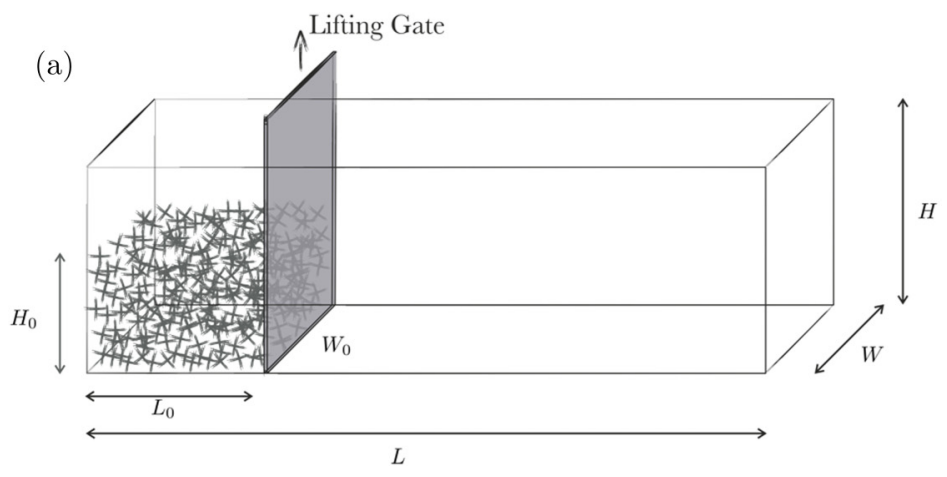

(b)

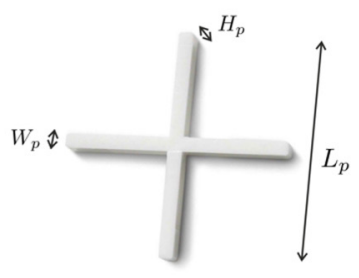

FIG. 4. Schematics of the experimental setup: (a) dam break apparatus and (b) shape of the particles.

on a separate optical table. Hence, the vibration of the gate coming to a halt does not affect the experiments.

Four light sources are placed at different angles to illuminate the particles. A high-speed camera (FASTCAM Mini UX100) is used in combination with a Carl-Zeiss Makro-Planar T* 50-mm $f / 2$ ZF.2 lens. Videos are captured at 250 frames/s and the imaging setup results in a pixel size of $\sim 0.43 \mathrm{~mm}$.

In each experiment, the initial granular pile has dimensions of $L_{0} \times H_{0} \times W_{0}$, where $L_{0}$ is the length, $H_{0}$ is the height, and $W_{0}$ is the width. All these parameters are changed in the experiments, resulting in 46 different experimental conditions, each repeated up to 8 times.

\section{B. Particles}

Cross-shaped plastic tile spacers (from Faithfull) are used as particles [see Fig. 4(b)]. The particles have a length of $L_{p}=27 \mathrm{~mm}$, with an arm width of $W_{p}=2 \mathrm{~mm}$, and thickness of $H_{p}=3 \mathrm{~mm}$. Hence, the volume of each particle is $0.312 \mathrm{~cm}^{3}$. The mass density of the particles is measured to be $770 \mathrm{~kg} / \mathrm{m}^{3}$.

We follow Balmforth and Kreswell [3] to characterize the friction properties of the particles. We find the bed friction angle (between acrylic and particle) to be $\delta=32^{\circ} \pm 5^{\circ}$ and the internal friction angle (between particles) to be $\theta=24^{\circ} \pm 2^{\circ}$. Compared to glass particles (cf. Ref. [3]), the particles used in the present study have a larger bed angle of friction but a similar internal friction angle.

\section{Numerical setup}

To numerically reproduce the experiments, we use the contact model with history described in Sec. II B, with no rolling friction as our particles are neither spheres, cylinders, nor ellipsoids. Our contact model is validated for systems of spherical particles, as this model has not yet been applied to nonellipsoidal particles in the literature. Our validation procedure is presented in Appendix B. For each simulation, we proceed as follows. First, we generate the same geometry as in the experimental apparatus. Then the particles are poured in the container with random initial positions until the system reaches a static state. At $t=0$, the gate is removed. The number of particles considered ranges from 1300 to 7000 and the values assigned to the contact model parameters are listed in Table I. The normal stiffness $k_{n}$ is computed to yield a maximum overlap distance $\delta_{\max }$ of about $1 \%$ of the radius of the sphere of equal volume, using an impact velocity corresponding to a free fall from a $30 \mathrm{~cm}$ height, i.e., $\sqrt{2 \times 9.81 \times 0.3} \approx 2.4 \mathrm{~m} / \mathrm{s}$. This leads to $\delta_{\max } \approx 5 \times 10^{-5} \mathrm{~m}$. In practice, this maximum overlap is very rarely reached, and the average overlap is orders of magnitude lower, typically between $0.1 \%$ and $0.001 \%$ of the radius of the sphere of equal volume. Note that the contact parameters in the tangential direction are not set following the procedure described in Sec. II C 2. We discuss the reasons why this procedure is not followed in Sec. V C. 
TABLE I. Particle properties and contact model parameters in the numerical simulations of nonconvex particles.

\begin{tabular}{lccc}
\hline \hline Name & Symbol & Value & Unit \\
\hline particle density & $\rho$ & 770 & $\mathrm{~kg} / \mathrm{m}^{3}$ \\
normal stiffness & $k_{n}$ & $4.5 \times 10^{5}$ & $\mathrm{~N} / \mathrm{m}$ \\
normal restitution coefficient & $e_{n}$ & 0.85 & \\
tangential stiffness & $k_{t}$ & $3 \times 10^{2}$ & $\mathrm{~N} / \mathrm{m}$ \\
tangential damping factor & $\gamma_{t}$ & $10^{3}$ & $\mathrm{~s}^{-1}$ \\
particle-particle static friction coefficient & $\mu_{\mathrm{c} \text {,part-part }}$ & 0.46 & \\
particle-wall static friction coefficient & $\mu_{\mathrm{c} \text {,part-wall }}$ & 0.62 & $\mathrm{~s}$ \\
time step & $\Delta t$ & $2.5 \times 10^{-6}$ & \\
\hline \hline
\end{tabular}

To ensure each case is treated in a systematic way during the postprocessing step, we determine quantities such as the final height and the final length using image-processing routines. In particular, a quantity that is not obvious to define is the final length of the granular assembly, also called runout distance $L_{\infty}$. We follow the same approach as Rakotonirina et al. [29] and define the runout distance as follows: Using a top view of the final configuration, the runout distance $L_{\infty}$ is the leftmost column of pixels with a fraction of white pixels (referred to as intensity of the pixel column) that is less than $10 \%$. Figure 5(a) shows a typical output of this postprocessing routine. The solid line corresponds to the pixel column intensity and the dashed line corresponds to the runout distance $L_{\infty}$. Given the uncertainty of this measurement, we provide error bars in Fig. 10 with a minimum value corresponding to the runout distance based on a $20 \%$ intensity threshold and a maximum value corresponding to the runout distance based on a $1 \%$ intensity threshold. Similarly, the initial and final heights of the granular assembly are measured using an image-processing routine that computes the average height of the pile along the width of the channel, shown in Fig. 5(b). The solid line corresponds to the average height and the dotted lines indicate the first and third quartiles, used to compute the error bars in Fig. 11.

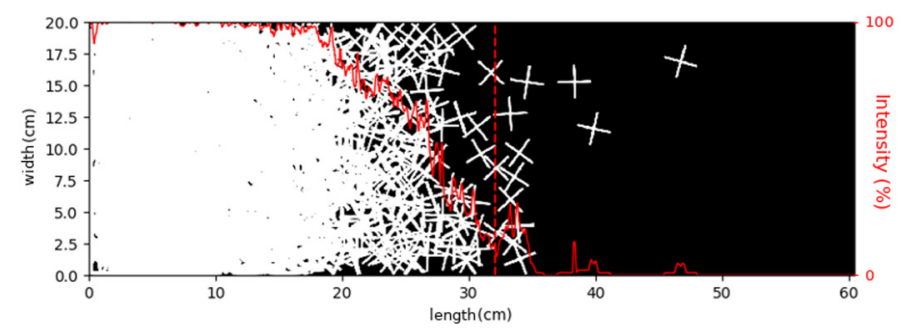

(a)

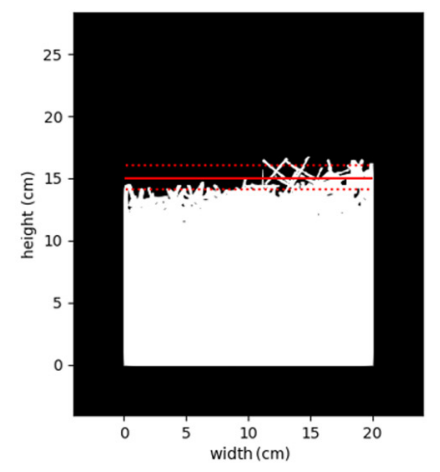

(b)

FIG. 5. (a) Example of a postprocessing output for the runout distance $L_{\infty}$ of a simulation, from a top view of the avalanche. The solid line corresponds to the intensity of a given pixel column (the maximum intensity corresponds to the top of the image and the minimum intensity to the bottom). The dashed line indicates the runout distance; in this case, $L_{\infty}=32 \mathrm{~cm}$. (b) Example of a postprocessing output for the final height $H_{\infty}$ of a simulation. The solid line indicates the average final height and the dotted lines show the first and third quartiles. For visual purposes, an example with a particularly wide interquartile range (IQR) is shown (a typical IQR is half the size of that shown on this figure). 
Finally, in order to quantitatively compare flow properties of nonconvex particles and spheres, we carry out numerical simulations of dam break avalanches of spheres using the same numerical setup and contact parameters as Girolami et al. [16]. In particular, we compute cases 1, 2, and 3 (each case is computed ten times corresponding to ten different initial microstructures) from Table 1 of [16] and we successfully reproduce results from Fig. 4 in [16], which shows very satisfactory agreement between numerical simulations of spherical particles and the experiments of Lajeunesse et al. [2]. In the following, we use these simulations to access data that are not reported in [16], such as the average velocity versus time.

\section{EXPERIMENTAL AND NUMERICAL RESULTS}

\section{A. Qualitative analysis}

Figures 6 and 7 present the experimental and numerical results of two relevant avalanche behaviors. For those two figures only, in order to visually compare the simulations with the experiments, a gate effect is included in the simulations, i.e., the particles in contact with the gate experience friction when the gate slides upward at a finite speed. The top-driven collapse, in Fig. 6, is by far the most common avalanching mechanism of our cross-shaped particles and is observed for all aspect ratios. During this collapse, the top part of the column falls first in a wavelike fashion. As particles are entangled, their fall is impeded by their neighbors, except for the top layer of particles which experiences less entanglement. As this top layer can fall freely off the granular column, a new layer of particles can repeat this process. This top-driven collapse is not observed for spherical or even convex-shaped particles $[2,3,16]$ : It is intrinsically linked to the entanglement of the cross-shaped particles. Sometimes, an initial microstructure leads to another avalanche behavior presented in Fig. 7. Unlike the top-driven collapse, the avalanche is triggered below the top layer of particles, around the midheight of the column. It results in the column bending forward during the first stage of the avalanche, before fully collapsing. This buckling behavior is found to be far less frequent than the top-driven collapse and is only observed for tall narrow columns as shown in Fig. 9.

It is observed experimentally and numerically that sometimes even the top layer of particles is too entangled to avalanche and the granular column remains stable after the opening of the gate. These entangled configurations are typical of nonconvex particles and pseudostable arches have been reported in the case of a hopper discharge of Schönhardt polyhedra by Govender et al. [33]. We find experimentally and numerically that for particular initial dimensions of the column, whether an avalanche occurs or the system remains stable is determined by the random initial configuration of the particles. We denote by intermittent this behavior where reproducibility is lost and show an example in Fig. 8. The intermittence is another difference of these entangled flows, as a granular dam break performed under the same conditions produces the same macroscopic outcome when convex particles are considered.

Figure 9 shows the experimental and numerical flow regime maps with respect to the initial length $L_{0}$ and initial height $H_{0}$. Each case was reproduced three to five times in order to assess its intermittence. In this figure, the transitions between flow regimes are not sharp and the purpose of these flow regime maps is to report qualitative trends rather than to provide accurate flow transitions. In our numerical simulations, the transition from the repeatable regimes to the intermittent regimes differs from the experiments at high aspect ratios. Indeed, it is found numerically that tall columns of initial height $H_{0}$ greater than $20 \mathrm{~cm}$ always result in an avalanche, while experimentally some cases were observed to be intermittent, for instance, $\left(L_{0}, H_{0}\right)=(12,25)$. This can be explained by a slightly inaccurate choice of parameters in Table I, and a discussion on the choice of tangential friction parameters is provided in Sec. V C. Nevertheless, we note that the numerical flow regime map agrees reasonably well with the experimental map, in particular in the region where buckling collapses occur as well as on the transition between the repeatable regimes and the intermittent regimes at low aspect ratios. 

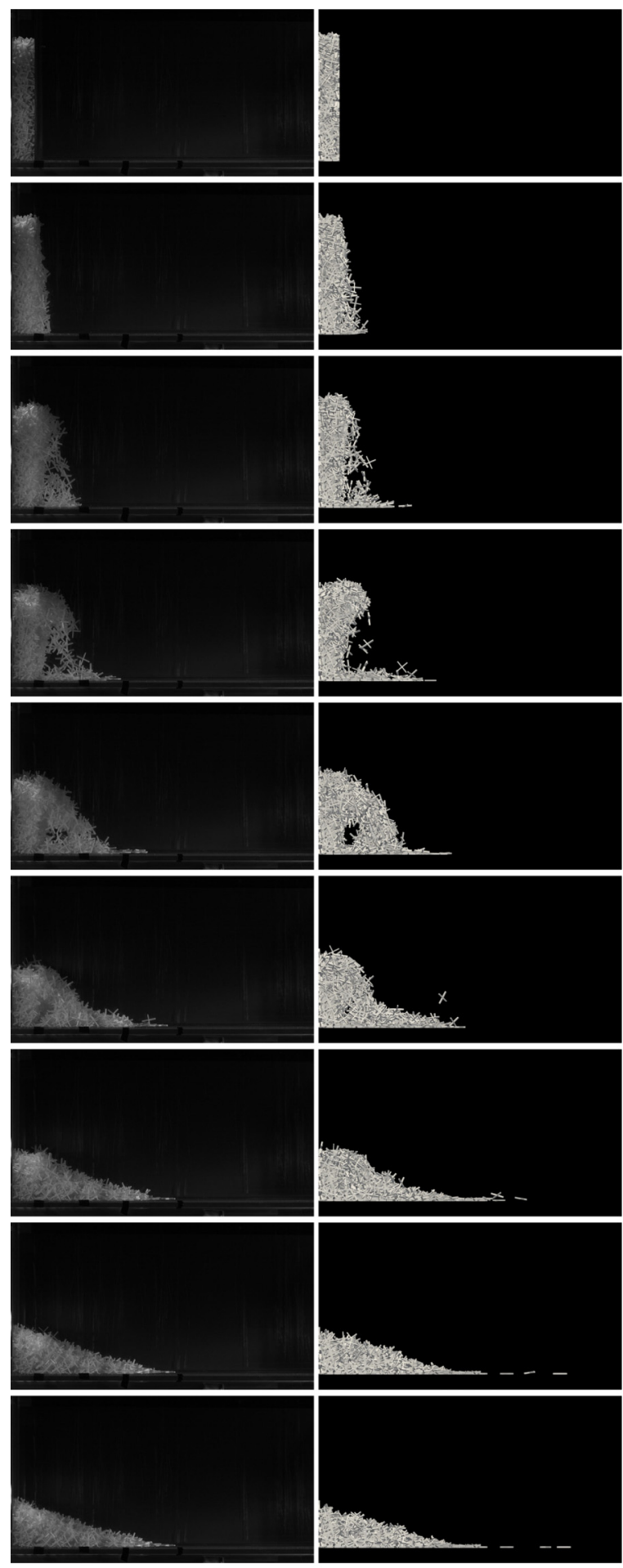

(a)

(b)

FIG. 6. Snapshots of a top-driven collapse with an initial aspect ratio $H_{0} / L_{0}=6$ from (a) experiments and (b) simulation. The time increment between each frame is $0.145 \mathrm{~s}$. Videos of this collapse are available in [47]. 


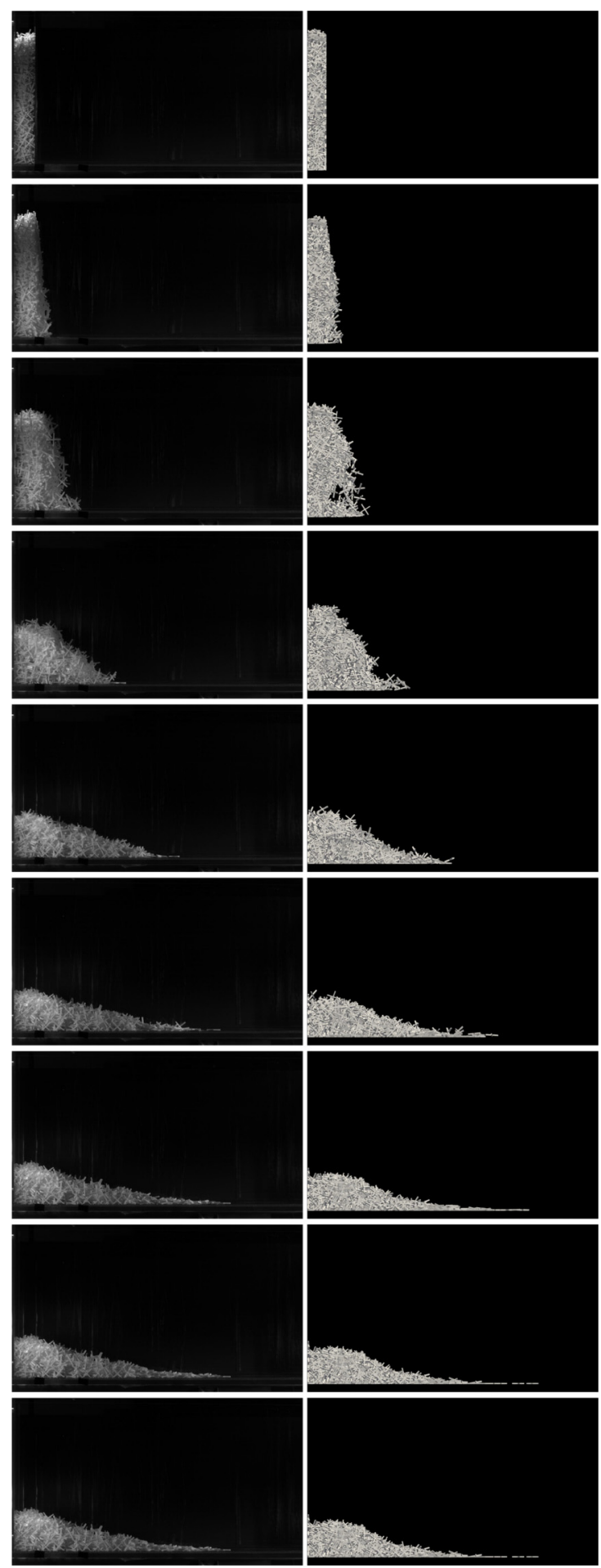

(a)

(b)

FIG. 7. Snapshots of a buckling collapse with an initial aspect ratio of $H_{0} / L_{0} \approx 7$ from (a) experiment and (b) simulation. The time increment between each frame is $0.12 \mathrm{~s}$. Videos of this collapse are available in [48]. 
(a)
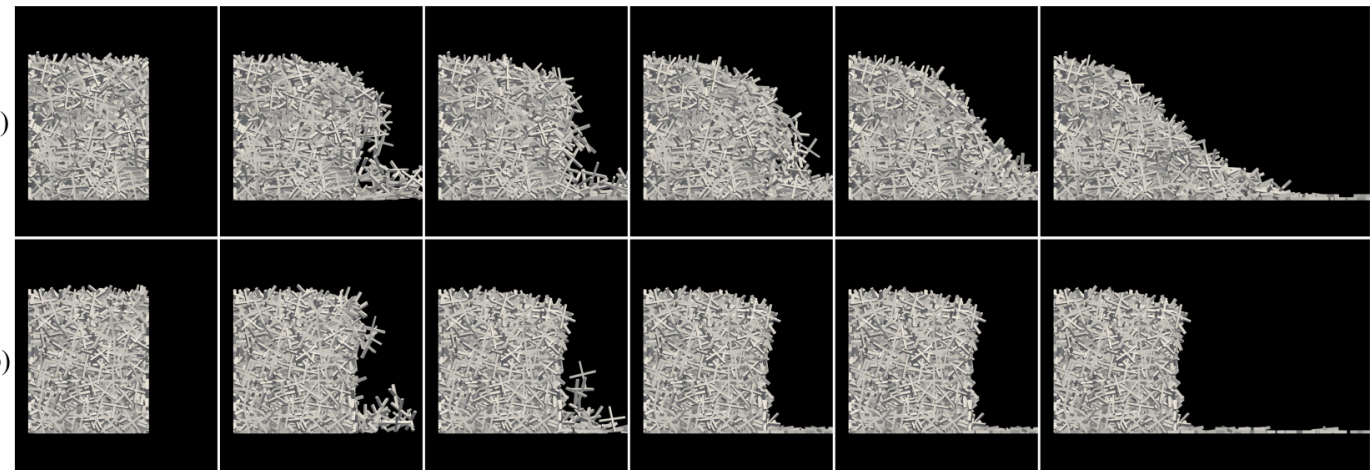

FIG. 8. Snapshots of (a) a simulated collapsing assembly and (b) a simulated stable assembly. The initial dimensions of both assemblies are identical; only the initial microstructure differs between these two intermittent cases. The frames are taken at the following times (from left to right): $t=0,0.30,0.60,0.90,1.20$, and $2.00 \mathrm{~s}$.

Aside from the intermittent regimes, we also recover the collapsed regime and the fractured regime that have already been reported for systems of spherical particles [2,3]. In the collapsed case, an avalanche occurs over the whole length of the granular assembly, resulting in a final height $H_{\infty}$ lower than the initial height $H_{0}$. In the fractured case, the initial length $L_{0}$ of the granular assembly is large enough such that some particles located to the left of a fractured line are not disturbed by the avalanche. In a fractured case, the final height $H_{\infty}$ of the granular assembly equals its initial height $H_{0}$.

\section{B. Macroscopic measurements}

\section{Runout distance and height ratios}

The runout distance $L_{\infty}$ and the final height $H_{\infty}$ of the avalanches are two easily accessed quantities that can be used to quantify to what extent avalanches of nonconvex particles differ from avalanches of convex particles, as well as to what extent our simulations agree with our

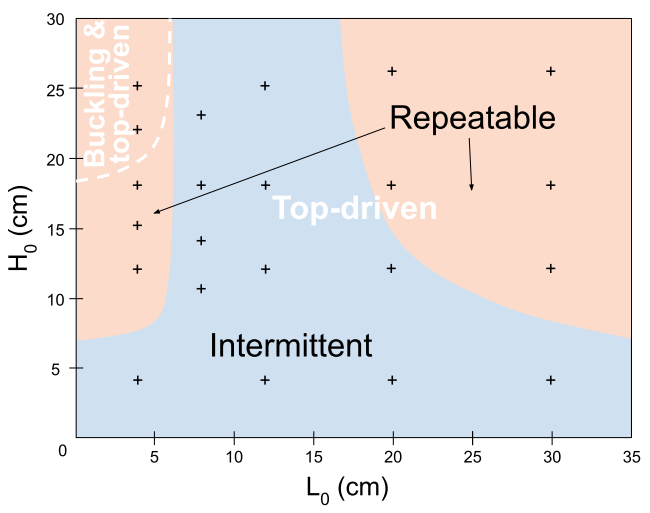

(a)

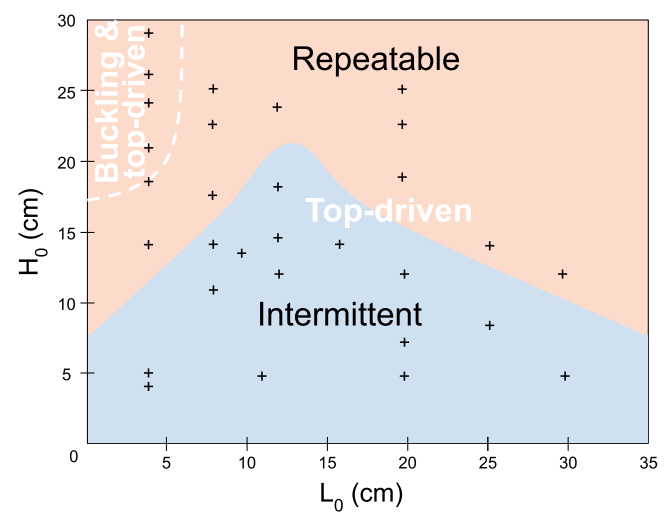

(b)

FIG. 9. Flow maps from (a) experiments and (b) simulations. Each cross represents an experiment or a simulation. Two flow regimes are identified, repeatable and intermittent, and two collapse behaviors are reported, top driven (observed in all configurations) and buckling (observed only for narrow columns). 


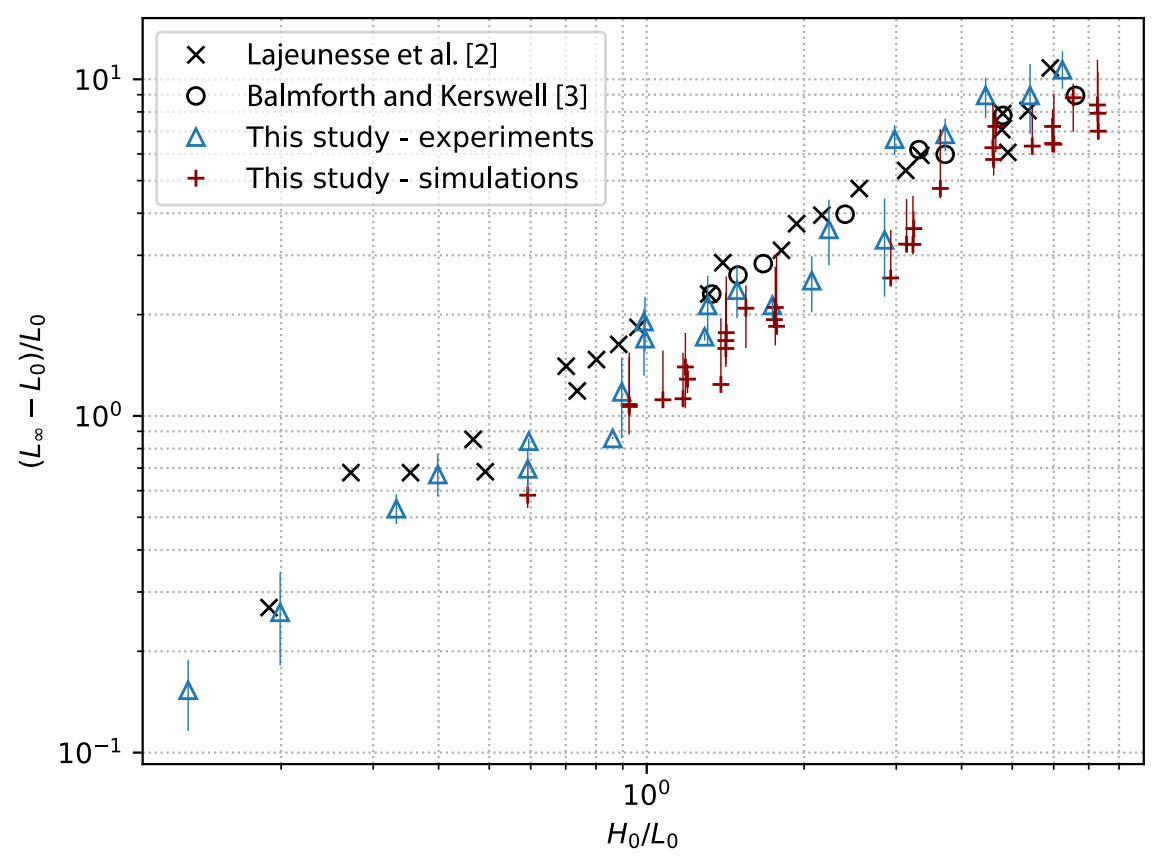

FIG. 10. Nondimensional runout distance $\left(L_{\infty}-L_{0}\right) / L_{0}$ as a function of the initial aspect ratio $H_{0} / L_{0}$. Error bars are depicted with vertical segments.

experiments. Figure 10 shows that the nondimensional runout distance $\left(L_{\infty}-L_{0}\right) / L_{0}$ as a function of the initial aspect ratio $H_{0} / L_{0}$ follows the same linear trend in the experimental and numerical cases. The error bars of the experimental and numerical data points largely overlap and the overall agreement between our simulations and our experiments is very satisfactory. This figure also includes experimental data from Lajeunesse et al. [2] and Balmforth and Kerswell [3], who carried out similar experiments with spherical particles. Figure 10 shows that, surprisingly, a very similar power law of the form $K\left(H_{0} / L_{0}\right)^{n}$ is followed by spherical and cross-shaped particles, even though the avalanche dynamics is very different. The power coefficient $n$ seems to be approximately equal to 1 for our cross-shaped particles, as found by Lajeunesse et al. [2] and Balmforth and Kerswell [3] for spheres or grit particles. The transition from $n \approx 1$ to $n \approx 2 / 3$ around $H_{0} / L_{0}=3$ reported by Lajeunesse et al. [2] for spheres is not very visible for our cross-shaped particles in Fig. 10. However, the analysis of the center of mass of the assembly reveals very clearly this slope transition around $H_{0} / L_{0}=3$ and indeed agrees with the measurements of Lajeunesse et al. [2], who considered initial aspect ratios of up to 20. The interested reader is referred to Appendix $\mathrm{C}$ for more details on this analysis. We also note that the prefactor $K$ is slightly lower for our cross-shaped particles as a signature of the shape and of the corresponding avalanche dynamics. Moreover, for $H_{0} / L_{0} \gtrsim 4$, the runout distance of all particle shapes, i.e., the spheres of Lajeunesse et al. [2], the grit particles of Balmforth and Kerswell [3], and our cross-shaped particles, almost matches, indicating that in more inertial regimes, the shape matters less.

Likewise, Fig. 11 shows the nondimensional height ratio $H_{0} / H_{\infty}$ as a function of the initial aspect ratio $H_{0} / L_{0}$ for our simulations and our experiments and also includes data obtained with grit particles by Balmforth and Kerswell [3]. Again, our simulations agree very well with our experiments and surprisingly again the cross-shaped particles follow the same trend as the grit particles, despite fundamentally different dynamics. 


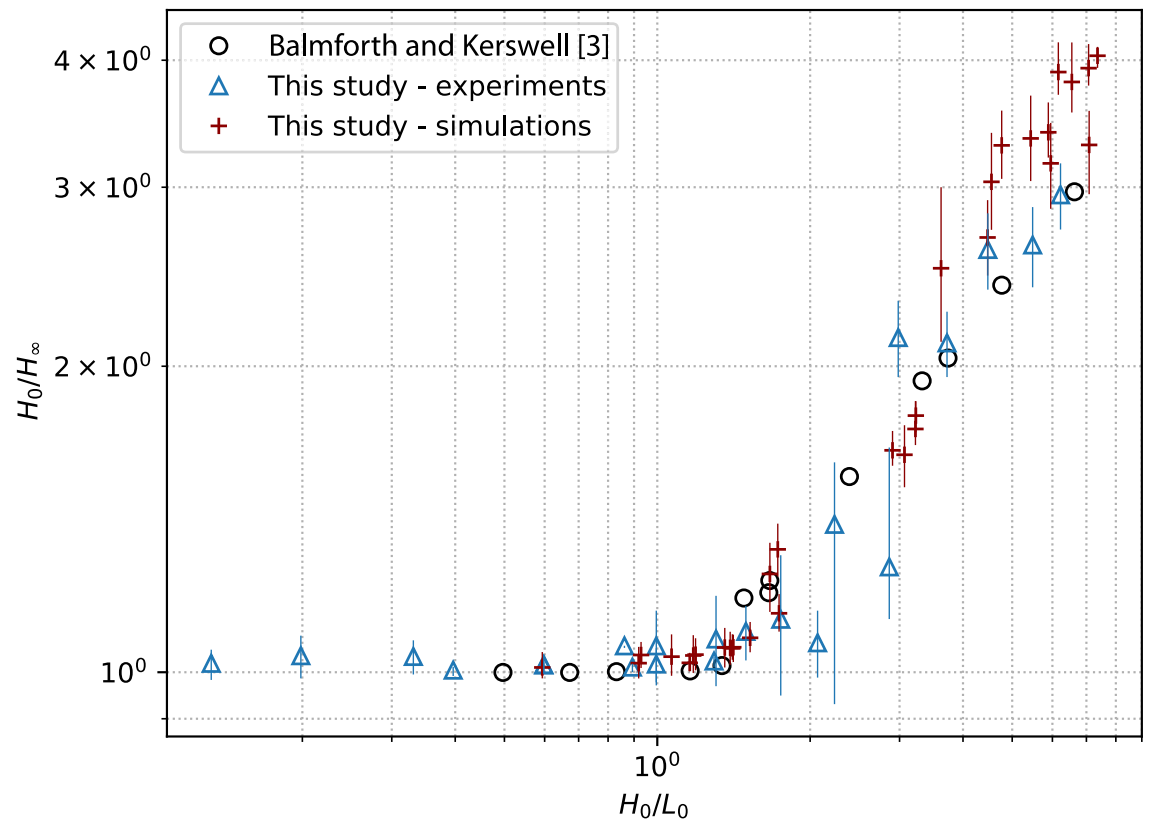

FIG. 11. Nondimensional height $H_{0} / H_{\infty}$ as a function of the initial aspect ratio $H_{0} / L_{0}$. Error bars are depicted with vertical segments.

\section{Characteristic time of collapse}

We quantitatively compare the time evolution of avalanches of crosses and spheres. Figure 12(a) shows the average nondimensional translational particle velocity $\tilde{v}_{\text {avg }}=v_{\text {avg }} / \sqrt{g H_{i}}$ versus the
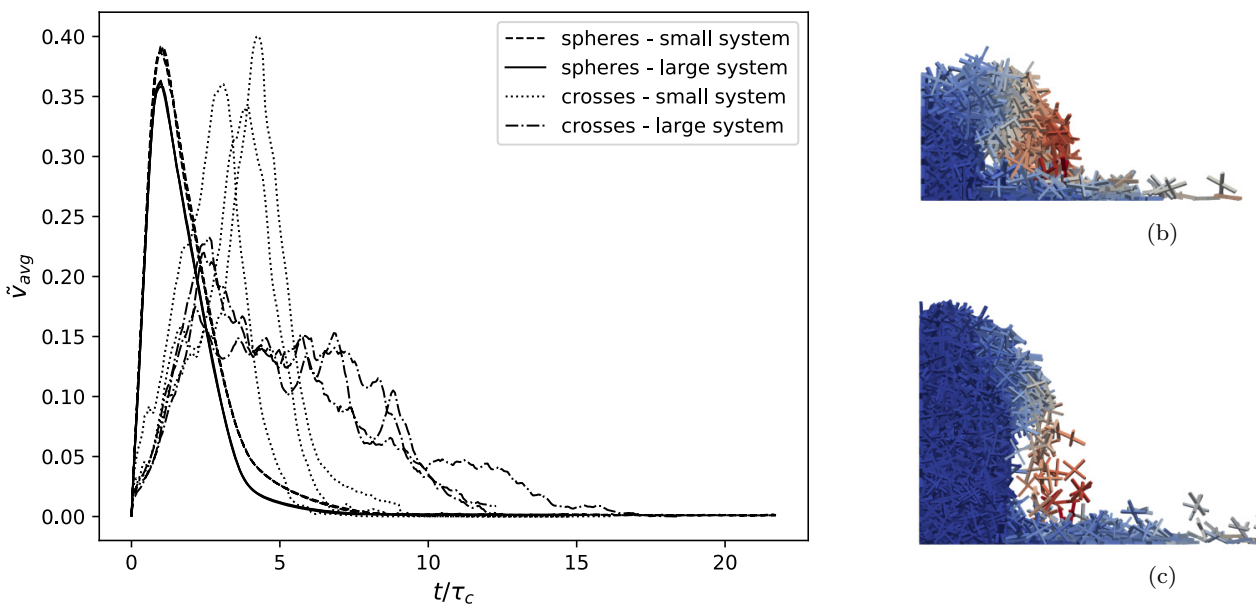

(b)

(a)

FIG. 12. (a) Comparison of the average nondimensional translational particle velocity versus nondimensional time for systems of spherical and cross-shaped particles in systems of initial aspect ratio $a=H_{0} / L_{0}=$ 3.2. Also shown are snapshots of the collapse of (b) a small and (c) a large system of crosses at $t=3 \tau_{c}$, colored by the norm of their translational velocity from dark blue (smallest) to red (largest). The small system has 3200 spheres or 1000 crosses and the large system 15000 spheres or 3000 crosses. 
nondimensional time $\tau_{c}=\sqrt{H_{i} / g}$ for avalanches of spherical particles and cross-shaped particles with an initial aspect ratio of 3.2. Small systems (3200 spheres or 1000 crosses) and large systems (15000 spheres or 3000 crosses) are considered and for each case three simulations with different initial microstructure are plotted in order to show reproducibility. On the one hand, the time evolutions of the velocity of small and large systems of spherical particles appear to be very reproducible, as the curves for each case superimpose very well. Moreover, the small and large systems behave similarly, with the average velocity reaching its maximum of 35\%-39\% of $v_{0}=\sqrt{g H_{i}}$ at $t=\tau_{c}$ and dropping below $5 \%$ of $v_{0}$ at $t \approx 4 \tau_{c}$. On the other hand, the velocity of cross-shaped particles is far less reproducible from one run to another. The small systems of crosses seem to follow a trajectory close to that observed with spheres, but delayed by two to four time units $\tau_{c}$, with a maximum velocity of $34 \%-39 \%$ of $v_{0}$. However, the large systems of crosses exhibit a very different trend: For each run the curve is flatter, its maximum is comprised between $18 \%$ and $22 \%$ of $v_{0}$ and occurs at $t \approx 2.5 \tau_{c}$, and the average velocity drops below $5 \%$ of $v_{0}$ at $t \approx 10 \tau_{c}$. Figures 12 (b) and 12(c) show snapshots of small and large systems of crosses at $t=3 \tau_{c}$. Particles are colored by the norm of their translational velocity. The main difference in the dynamics of the collapse of small and large systems of crosses is that small systems are narrow enough (less than the size of two particles) for the whole column to bend forward during the top-driven collapse. For instance, we see in Fig. 12(b) that the highest point of the column is located far away from the left wall, close to the previous location of the gate. In contrast, large systems (of width greater than the size of three particles) do not bend forward during the top-driven collapse: Fig. 12(c) shows how the highest point of the granular assembly is close to the left wall. Thus, the rate of particles flowing down the granular column is lower than that of smaller systems where the whole column bends forward. As a result, the maximum average velocity is lower and the collapse time is greater for large systems of crosses than for small systems of crosses.

A more striking time-dependent behavior inherent to entangled particles is the case of staggered collapses, which have been observed experimentally and numerically to appear in the case of initial aspect ratios corresponding to the intermittent and fractured regimes. In an unrepeatable way, some initial microstructures lead to collapses that come to nearly a halt for a few $\tau_{c}$ before continuing until fully avalanched, as shown in Fig. 13. It is important to note that the two curves in Fig. 13 correspond to two identical simulations with the exception of different initial microstructures. A partial staggered collapse is often observed for those regimes, i.e., the collapse is three dimensional and one region in the width direction halts its avalanche for up to a few seconds (a very long time for these kinds of granular systems) before fully collapsing.

This quantitative analysis in time suggests that the free fall characteristic time $\tau_{c}$, although relevant in the case of avalanches of spherical particles [2,3], does not describe collapses of entangled cross-shaped particles well. As such, defining a relevant timescale $\tau_{c}$ for granular collapses of entangled cross-shaped particles appears to be a complex matter and is left for future studies.

\section{Microstructure analysis}

We now extract information about the contact force network from our DEM simulations. This type of information cannot be measured experimentally. Figure 14 shows the contact network for a column of 15000 spheres (41 971 contacts in total) and a column of 12000 crosses ( 98939 contacts in total). The corresponding average coordination number is therefore $2 \times 41971 / 15000 \approx 5.6$ for spheres and $2 \times 98939 / 12000 \approx 16.5$ for crosses. The much larger average coordination number of crosses compared to that of spheres is expected as the arms of the crosses allow one to reach more neighboring particles located farther away. In Fig. 14, each segment represents a branch vector, i.e., each segment links the centers of mass of two contacting particles, and is colored according to $F_{c} /\left\langle F_{c}\right\rangle$, where $F_{c}$ is the contact force magnitude and $\left\langle F_{c}\right\rangle$ is the average contact force magnitude in the whole granular assembly. While it is not convenient to interpret Fig. 14(a) where all the branch vectors are shown, we plot in Fig. 14(b) the branch vectors associated with a contact force magnitude that is larger than 5 times the average contact force magnitude. Figure 14(b) clearly highlights that 


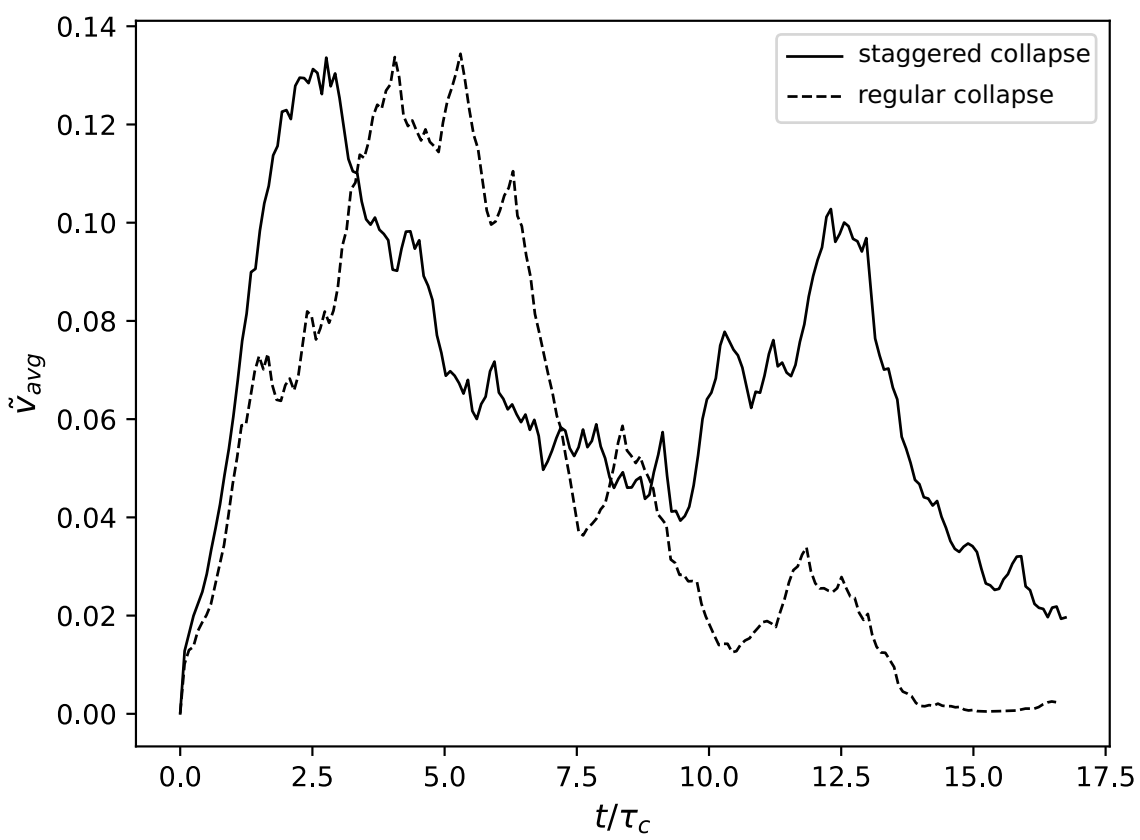

FIG. 13. Comparison of the average nondimensional translational particle velocity versus nondimensional time for a staggered collapse (solid line) and a regular collapse (dashed line) in the case of an initial aspect ratio $a=H_{0} / L_{0}=1.75$. The only difference between the two collapses is the random initial packing.

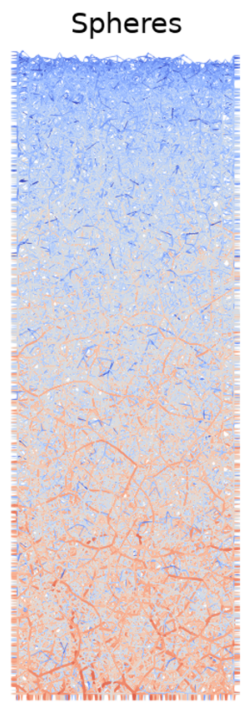

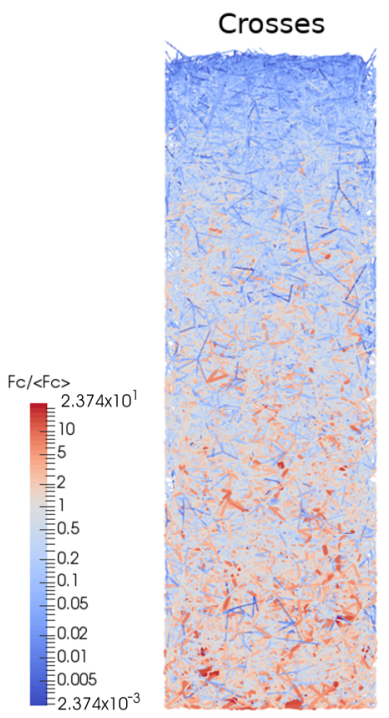

(a)
Spheres

Crosses
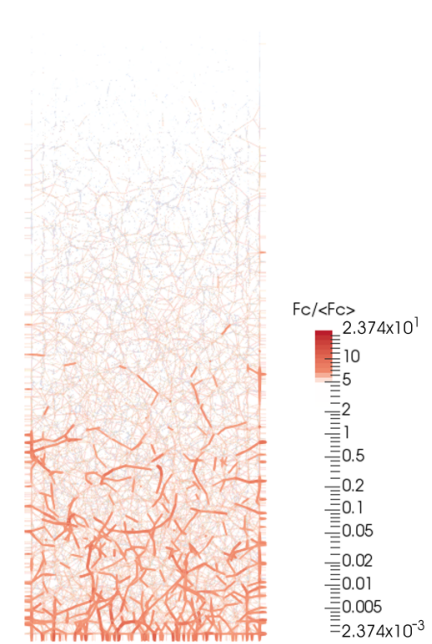

(b)

FIG. 14. Visualization of the contact network in a granular column composed of spheres and crosses. Each segment links the centers of mass of two contacting particles, and its color and width vary according to the magnitude of the total contact force magnitude $F_{c}$ divided by the average contact force magnitude $\left\langle F_{c}\right\rangle$. In (a) all contacts are shown, while in (b) only the contacts associated with a force magnitude greater than five times the average force magnitude are plotted. 


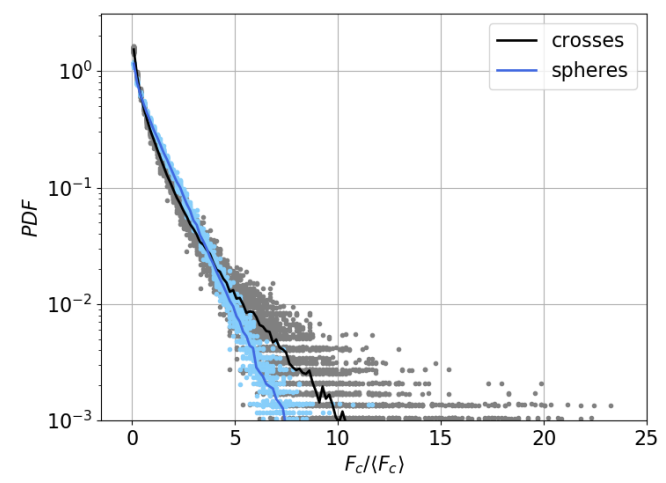

(a)

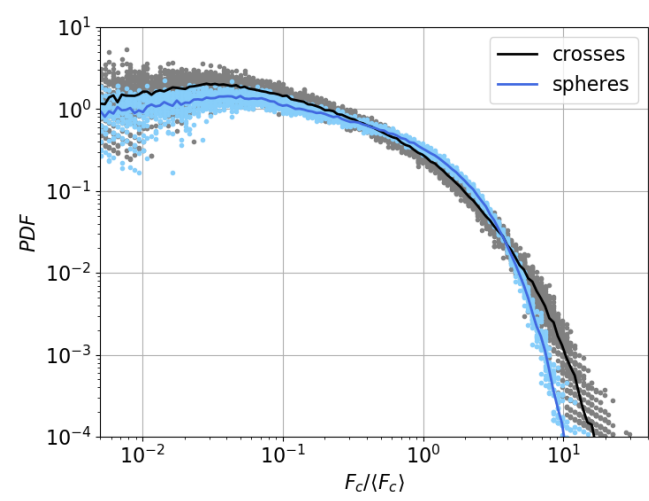

(b)

FIG. 15. Probability density function of the contact force magnitude $F_{c}$ divided by the average contact force magnitude $\left\langle F_{c}\right\rangle$ just before the gate is opened, in (a) linear scale and (b) logarithmic scale, in the cases of assemblies of crosses (black) and spheres (blue).

the cross-shaped particle assembly features many more and more homogeneously distributed large forces than the spherical particle assembly. Indeed, Fig. 14 shows that large contact forces in the spherical particle assembly are located along what is commonly referred to in the literature as a backbone, i.e., a subnetwork that features a small number of connected large contact forces, also called force chains, and that supports most of the external stress [49]. This prominent backbone subnetwork is not visible in the cross-shaped particle assembly.

\section{Probability density functions of contact forces}

We analyze the probability density function (PDF) of the contact force magnitude $F_{c}$ divided by the average contact force magnitude $\left\langle F_{c}\right\rangle$ in our granular assemblies. In Fig. 15 we compare the PDFs of $F_{c} /\left\langle F_{c}\right\rangle$ just before opening the gate in granular assemblies of crosses to that in granular assemblies of spheres. Each solid line corresponds to the average over $N$ simulations and each dot represents a data point, i.e., a contact force magnitude in one of the $N$ simulations. We take $N_{\text {spheres }}=30$ and $N_{\text {crosses }}=60$. In Figs. 15(a) and 15(b) the average PDF of $F_{c} /\left\langle F_{c}\right\rangle$ in the assembly of crosses is considerably broader than that in the assembly of spheres. The largest $F_{c} /\left\langle F_{c}\right\rangle$ in the assembly of crosses is 32 , while it is only 14 in the assembly of spheres. Forces in the range $F_{c} /\left\langle F_{c}\right\rangle \gtrsim 4$ are more probable in the assembly of crosses than in the assembly of spheres, in accordance with our qualitative observations of Fig. 14(b). While the large forces are more homogeneously distributed in the assembly of crosses than in the assembly of spheres (no prominent backbone; see Sec. IV C), consideration of the whole force range and the coordination number indicates that assemblies of crosses are more connected but also less homogeneous. Figures 15(a) and 15(b) agree well with previous works published in the literature. Saint-Cyr et al. [50] also reported broader PDFs of $F_{c} /\left\langle F_{c}\right\rangle$ in the case of nonconvex particles compared to the case of disks in their two-dimensional study of sheared particles with various degrees of concavity. Estrada et al. [51] studied disk-shaped particles and modeled their entanglement by inhibiting rotational motion. Their PDFs of $F_{c} /\left\langle F_{c}\right\rangle$ (see Figs. 14 and 15 in their study) are very similar to our PDFs plotted in Figs. 15(a) and 15(b). A notable difference with [51], however, is that the force network of their entangled granular assemblies qualitatively shows a well-defined backbone while our assemblies of cross-shaped particles do not, as previously discussed in Sec. IV C. We attribute this discrepancy to two factors: (i) Due to no gravity in [51] versus gravity in our simulations, our simulations cannot show "floating particles," i.e., particles with no contact with any neighboring particle, that promote the formation of a backbone and (ii) not only do our high-aspect-ratio particles reach neighbors located farther away than their disk-shaped counterpart, but their nonconvexity allows two particles 


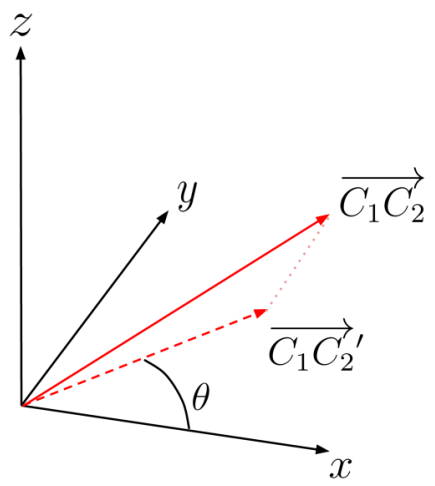

FIG. 16. Definition of the branch vector projected onto the vertical $x z$ plane and of the angle $\theta$ it forms with the horizontal direction.

to contact at multiple points, therefore dividing a branch vector into several weaker branch vectors. To summarize, the PDFs of $F_{c} /\left\langle F_{c}\right\rangle$ shown in Figs. 15(a) and 15(b), the absence of backbone in Fig. 14(b), and the observed intermittent regime which highlights the high stability of assemblies of crosses show that assemblies of crosses do not necessitate the presence of a backbone to resist an exerted stress load. In a broader sense, this conclusion suggests that in granular assemblies of entangled particles both the diffuse subnetwork of relatively large contact forces and the subnetwork of small forces play an important role in resisting the exerted stress load [51]. We plot in Appendix D similar comparisons of PDFs of $F_{c} /\left\langle F_{c}\right\rangle$ for (i) assemblies of crosses about to collapse in a top-driven fashion versus in a buckling fashion and (ii) assemblies in the intermittent regime about to collapse versus remaining stable. In both cases, the PDFs of $F_{c} /\left\langle F_{c}\right\rangle$ superimpose very well, suggesting that the distribution of the contact force magnitude is not relevant in determining the collapse outcome.

\section{Fabric anisotropy}

In granular dynamics, fabric anisotropy refers to the preferred directions of branch vectors $\overrightarrow{C_{1} C_{2}}$ connecting the centers of mass of two contacting particles. While the branch vector direction coincides with that of the normal contact force in the case of spheres, it does not in the general case of nonspherical particles. In the following, we restrict the analysis to the branch vectors projected onto the $x z$ vertical plane normal to the width of the channel and we define the angle $\theta$ formed by the horizontal direction $x$ and the projected branch vector ${\overrightarrow{C_{1}} C_{2}}^{\prime}$, as shown in Fig. 16. Figure 17 compares the average fabric anisotropy of assemblies of spheres, crosses, and coin-shaped particles. The collapse behavior of the assemblies of crosses considered in Fig. 17 belongs to various regimes: top driven, buckling, intermittent stable, and intermittent collapsed. Undesirable wall effects are minimized as follows: (i) The length $L_{0}$ of the initial column is chosen to be greater than 5 times the particle circumscribed diameter [except in Fig. 17(b), where the aspect ratio is required to be very high in order to observe buckling collapses as shown in Fig. 9] and (ii) contacts with the walls are excluded from the analysis.

Figure 17(a) reveals that the preferred direction of the branch vectors in the assembly of spheres is the vertical direction, as expected for a random loose packing of spheres under gravity. Conversely, the preferred direction of the branch vectors in the assembly of crosses is the horizontal direction, i.e., orthogonal to the direction of gravity. This result is simply due to the large aspect ratio of crosses and the fact that the stable position of a cross onto a horizontal plane is to lie flat and align its arms with the horizontal direction in order to minimize its gravitational potential energy. Consequently, in the absence of wall effects, the average angular position of crosses has a tendency to get closer to $0^{\circ}$ and $180^{\circ}$ as the aspect ratio of the cross-shaped particles increases. This can be generalized to other shapes with a large aspect ratio. To support this claim, we also plot in Fig. 17(a) the fabric 


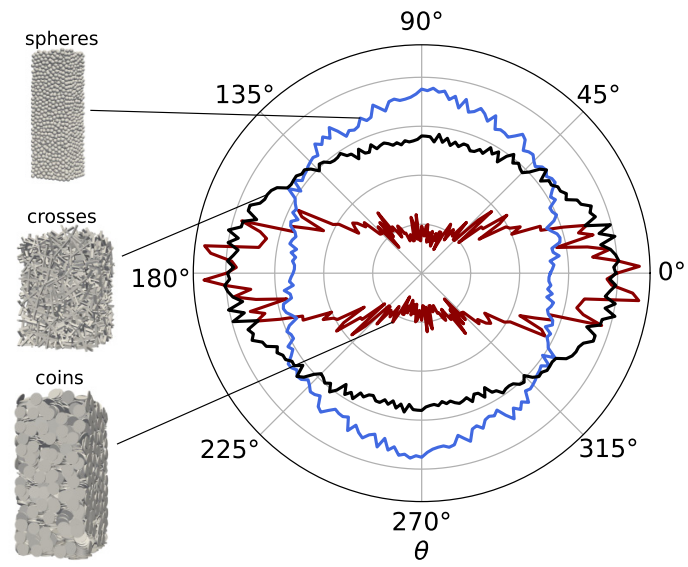

(a)

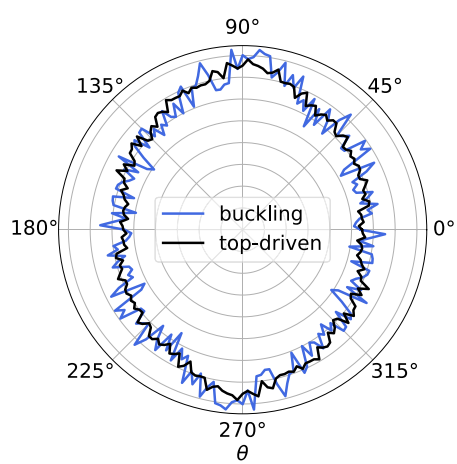

(b)

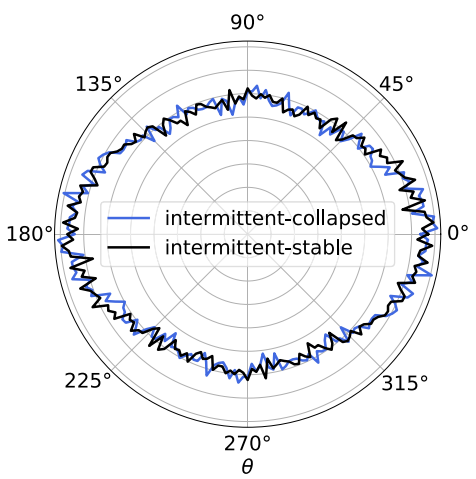

(c)

FIG. 17. Average fabric anisotropy of various granular assemblies prior to the opening of the gate. (a) Comparison of the fabric anisotropy of spheres (averaged over 30 simulations) to crosses (averaged over 60 simulations) and coins (only one simulation shown); the curve for coins has been scaled down for readability. (b) Comparison of the fabric anisotropy of crosses about to collapse in a top-driven fashion (averaged over 50 simulations) to crosses about to collapse in a buckling fashion (averaged over 6 simulations). (c) Comparison of the fabric anisotropy of crosses in the intermittent regime about to collapse (averaged over 18 simulations) to remaining stable (averaged over 26 simulations).

anisotropy of coin-shaped particles with a thickness and a circumscribed diameter identical to those of crosses. Since these convex coin-shaped particles cannot entangle with neighboring particles the way crosses do, their fabric anisotropy plotted in red in Fig. 17(a) reveals an even stronger tendency to lie flat and align with the horizontal direction than that of crosses.

Figure 17(b) shows the fabric anisotropy of assemblies of crosses leading to buckling collapse and to top-driven collapse. In this case the preferred direction of the branch vectors is vertical both in the buckling case and in the top-driven case. This result is simply a consequence of the rather small $L_{0}$ considered in these cases in order to be able to investigate the large initial aspect ratio of the column while keeping the total number of particles in the system at a tractable level. In narrow columns with significant wall effects, crosses are constrained by the lateral walls to align their arms with the vertical direction. Disappointingly, the fabric anisotropy of assemblies of crosses leading to buckling collapse and of assemblies of crosses leading to top-driven collapse almost perfectly overlap. We also compare the fabric anisotropy of assemblies in the intermittent regime about to collapse (named intermittent-collapsed) to remaining stable (named intermittent-stable) in Fig. 17(c). This plot does not reveal any marked difference either. The intermittent-collapsed 


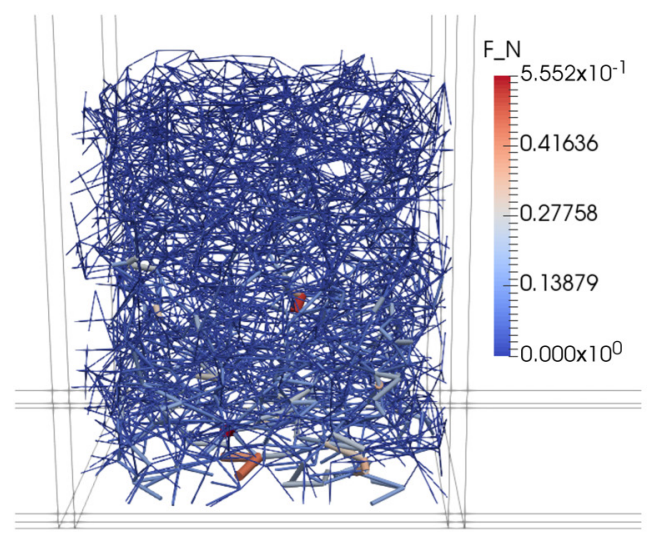

(a)

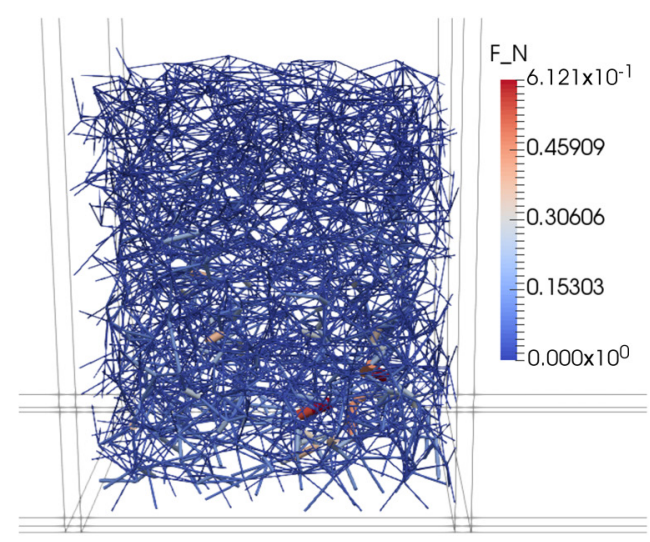

(b)

FIG. 18. Visualization of the force network of two intermittent simulations. Each segment links a contact point to the center of mass of a particle and is colored according to the magnitude of the normal component of the contact force. (a) The opening of the right gate leads to a granular collapse. (b) The opening of the right gate leads to a stable assembly.

fabric anisotropy and the intermittent-stable fabric anisotropy almost perfectly overlap, as in the comparison between buckling collapse and top-driven collapse.

At that stage, we conclude that the fabric anisotropy and the additional PDFs of $F_{c} /\left\langle F_{c}\right\rangle$ presented in Appendix D do not seem to be proper descriptors of the microstructure able to reveal salient features that could distinguish the various collapse behaviors: top driven versus buckling and intermittent-stable versus intermittent-collapsed. In Sec. V A we attempt to distinguish intermittentcollapsed from intermittent-stable through examining the average coordination number and the Janssen effects on the walls.

\section{DISCUSSION}

\section{A. Analyzing the intermittent regime with DEM simulations}

In this section we investigate the intermittent regime using our DEM simulations. Figure 18 presents a three-dimensional view of the force network of configurations that collapse [Fig. 18(a)] and remain stable [Fig. 18(b)]. No qualitative discrepancy in the contact density nor the force magnitude can be visually noted. We could speculate that in the stable case [Fig. 18(b)] the largest contact force is located close to the gate, possibly reinforcing the stability of the granular assembly in this area. However, this does not hold for two reasons: (i) This particular stable case also remains stable when the left gate opens and (ii) some asymmetrical assemblies similar to Fig. 18(b) result in granular collapses. Similarly, we could note the small difference in the maximum force magnitude between the cases in Figs. 18(a) and 18(b), but this maximum force magnitude is reversed for other simulations and cannot be used as a determinant factor to predict a granular collapse.

Using a more systematic approach to investigate intermittence, we compute for each case a number of quantities such as the average coordination number, the Janssen effect on the walls, and number of contact points with the bottom wall, with the lateral walls, and with the gate. The raw data are presented in Appendix E. Surprisingly again, no trend is found in any of those average quantities, whether the whole granular assembly is considered or whether the analysis is restricted to subregions such as the region close to the gate and the region close to the top of the gate. We speculate that the stability of the assembly is governed by finer properties of the force network such as local clusters, i.e., subregions of densely entangled particles that are not well connected to the rest of the system, or the presence of force cycles, which tend to increase rigidity 
(a)
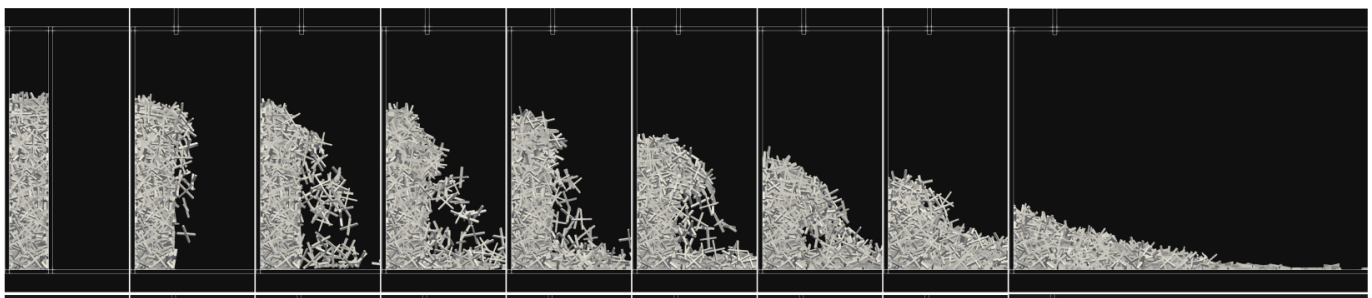

(b)
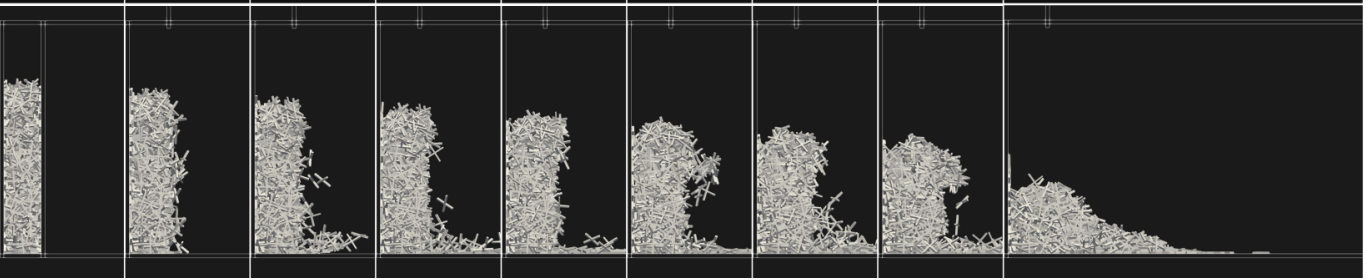

FIG. 19. Snapshots of simulations (a) with and (b) without memory in the contact model. The frames are taken at the following times (from left to right): $t=0,0.15,0.30,0.45,0.60,0.75,0.90,1.05$, and $3.00 \mathrm{~s}$.

of granular assemblies. Investigating granular assemblies from the perspective of network analysis is growing in interest [52], but it remains a difficult task and it is in most cases still restricted to systems of disk-shaped (sometimes spherical) particles. It is far beyond the scope of this paper to investigate a reliable network analysis for three-dimensional high aspect ratios and nonconvex particles.

\section{B. Importance of the contact model with memory}

Since the implementation of the contact model with memory is quite tedious but necessary, it is interesting to compare the results obtained with and without memory. Figure 19 shows the qualitative discrepancies of two simulations with memory [Fig. 19(a)] and without memory [Fig. 19(b)] and with the same initial microstructure. When the memory term is included in the contact model, we can see that the height of the column is decreasing because particles fall off the top of the pile. Conversely, when the memory is not taken into account in the contact model, the height of the column decreases faster while the number of particles released from the top is lower. Indeed, without memory the tangential friction vanishes where the relative velocity approaches zero: As a result, the granular column slowly deforms and overpacks at the bottom of the pile, while it should remain static in that region of space. This unphysical behavior was noted by Govender et al. [33]: In their study (Fig. 16 therein), they commented on the slow deformation of a simulated arch of nonconvex particles and noted that it does not match experimental data and that it even leads to the unphysical discharge of particles. In light of Fig. 19, we can confidently state that this unphysical deformation is the result of the absence of a memory term in the tangential friction force in their simulations. Figure 19 demonstrates that this memory term is of tremendous importance in order to numerically reproduce static configurations or dynamic configurations that include static subsystems.

\section{Choice of contact model parameters for nonspherical bodies}

The tangential contact model parameters listed in Table I are not obtained via the procedure provided in Sec. II C 2. This is because for reasonable values of the normal and tangential energy restitution coefficients $e_{n}$ and $e_{t}$, the avalanche never occurs and the pile of particles remains stable at all times for all aspect ratios. We discuss below why this procedure did not give satisfactory results when applied to our system of cross-shaped particles. 


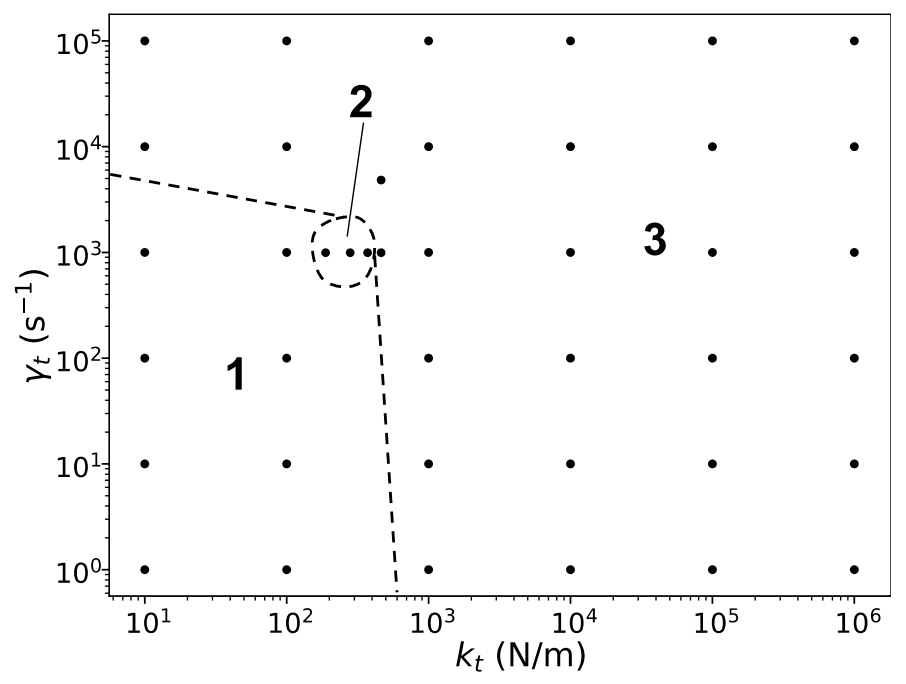

FIG. 20. Variation of the flow behavior with respect to the tangential parameters. Each dot corresponds to a simulation with specific tangential parameters $\left(k_{t}\right.$ and $\left.\gamma_{t}\right)$. Region 2 correctly reproduces experiments. Region 1 leads to avalanches that collapse like convex particles, i.e., the base of the pile drives the motion. Region 3 leads to fully entangled systems that do not collapse.

First, we span a wide range of values for the tangential parameters and report the type of behavior that arises. Values of $k_{t}$ range from $10^{1}$ to $10^{6} \mathrm{~N} / \mathrm{m}$ and values of $\gamma_{t}=\eta_{t} / 2 m_{i j}$ range from $10^{0}$ to $10^{5} \mathrm{~s}^{-1}$. The results are presented in Fig. 20. We can see that only a narrow region of the space formed by possible values of $k_{t}$ and $\gamma_{t}$ leads to a physical output of the DEM simulations. This region corresponds to $k_{t} \approx 3 \times 10^{2} \mathrm{~N} / \mathrm{m}$ and $\gamma_{t} \approx 10^{3} \mathrm{~s}^{-1}$.

Then we observe from Eqs. (16)-(19) that once a maximum overlap distance $\delta_{\max }$ is set, the only two variables the tangential parameters $k_{t}$ and $\eta_{t}$ are depending on are $e_{n}$ and $e_{t}$. For this analysis we are interested in orders of magnitude only; therefore, we assume that the distances between the contact point and the center of mass of the cross-shaped particles are always the same and are equal to the radius of the sphere of the same volume $R_{s}$. Figure 21 shows plots of $k_{t}$ and $\gamma_{t}$ for $e_{n}$ and $e_{t}$ existing in $(0,1)$. This figure shows how $k_{t}$ tends to zero extremely slowly when $e_{n} \rightarrow 0$. From

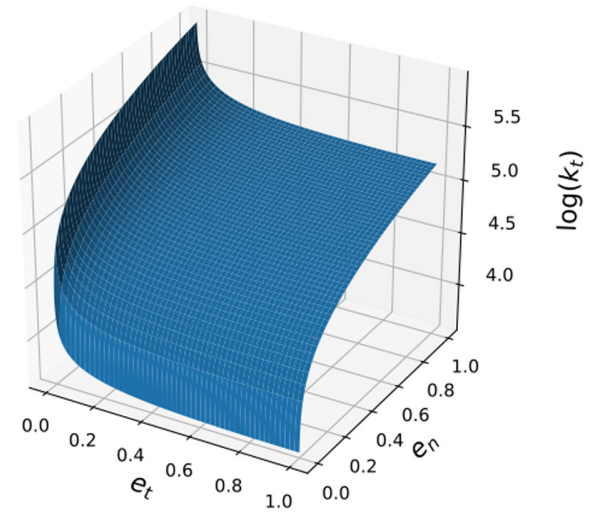

(a)

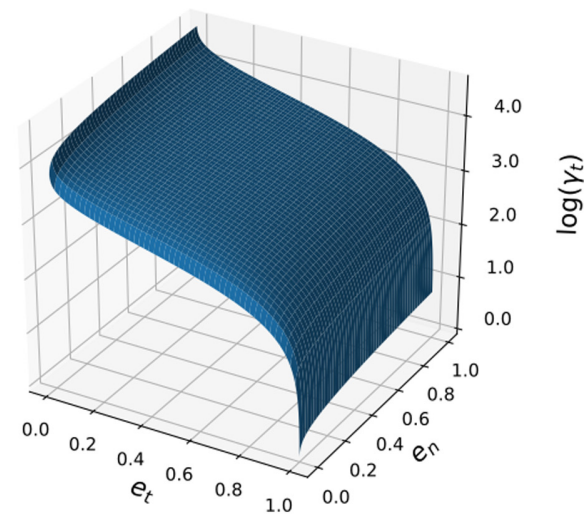

(b)

FIG. 21. Surface plots of the tangential parameters (a) $k_{t}$ and (b) $\gamma_{t}=\frac{\eta_{t}}{2 m}$ for $e_{n}$ and $e_{t}$ varying from 0 to 1 . 
Eqs. (16) and (18) we deduce the behavior of $k_{t}$ for $e_{n} \rightarrow 0$ and $e_{t} \rightarrow 1$ :

$$
k_{t} \underset{\substack{e_{n} \rightarrow 0 \\ e_{t} \rightarrow 1}}{\sim} \frac{C}{\ln \left(e_{n}\right)^{2}} \quad \text { with } C=\frac{\left(\pi v_{\max } / \delta_{\max } e\right)^{2}}{\frac{1}{m_{i j}}+2 \frac{R_{s}^{2}}{I_{s}}} .
$$

In our case, since $C \approx 2 \times 10^{5} \mathrm{~N} / \mathrm{m}$, this implies that $e_{n}$ must be of the order of $10^{-11}$ to reach $k_{t} \approx 3 \times 10^{2} \mathrm{~N} / \mathrm{m}$. Such a low value of the energy restitution coefficient is of course strongly unphysical, since it would imply that a particle impacting a plane bounces back with a velocity $10^{11}$ times smaller than the impact velocity.

At this point, we recognize that even though this procedure has been successfully applied to spherical shapes and ellipsoids [30], it cannot be used for particle shapes that differ significantly from spheres, such as the nonconvex and angular particles considered in this study. No procedure has been successfully derived to determine methodically the tangential contact parameters of complexshaped particles. In fact, Landauer et al. [10] face the same difficulties in choosing meaningful friction parameters and conclude that "it is impossible to use DEM simulations as a plug-and-play tool to predict particle packing and flow properties." As a result, the community keeps relying on calibration studies or on trial and error. Investigating further a reliable, physically based method or providing calibration guidelines [53] to set contact model parameters in DEM simulations of arbitrary-shaped particles would be of great interest in future works.

\section{Analogy with other stable granular assemblies}

Entanglement is not the only mechanism that can lead to stable piles; such stable granular columns have been reported in the case of cohesive particles [54] and fluid-saturated particles [55]. In [54] Abramian et al. conducted discrete and continuous two-dimensional simulations of the slumping of a column of cohesive particles and observed stable and collapsed regimes. In [55] Bougouin et al. examined the problem of the slumping of a fluid-saturated column of particles. In their study, varying the "column" Bond number, which compares gravity to capillary effects, also leads to stable and collapsed regimes. The authors carried out a dimensional analysis that captures very well the transition between the observed regimes. It is important to stress that in order to observe stable granular assemblies, a stabilizing mechanism at the particle scale is required. While in the case of Bougouin et al. (Abramian et al.) the capillary (cohesive) force plays the role of the stabilizing mechanism, in our study the stabilizing effect comes from the inhibition of particle displacement resulting from the entanglement of the particles with their neighbors. In a future work, we might attempt to design a column Bond number for entangled granular media and carry out a dimensional analysis similar to that of [55] in order to capture the transitions between the observed regimes. As this column Bond number would be the ratio of the gravitational force over the stabilizing force, it would require including a measure of the aforementioned displacement inhibition due to the presence of entangled neighbours. In a first approximation, this column Bond number could be a function of a mean entanglement number that would depend on the geometric properties of the particles. This entanglement number may be defined following the approach of Gravish et al. [9], i.e., as the number of neighbors crossing a portion of a plane specific to the shape of the considered particles. In the case of our cross-shaped particles, it would likely be a disk of diameter $L_{p}$ and located in the plane formed by the arms of the crosses. Then the entanglement number would be averaged over all particles in the column and treated as a particle feature in the dimensional analysis.

\section{CONCLUSION}

In this study we performed granular dam break experiments and simulations of angular nonconvex particles with high aspect ratios. We reported a wide range of flow regimes and dynamic behaviors, such as the intermittent regime and two collapsing dynamics: the top-driven collapse 
and the buckling collapse. Notable differences in the collapses of the considered cross-shaped particles with respect to spheres include a strong three-dimensionality of the avalanche, the absence of repeatability of most behaviors, the presence of staggered collapses, and the irrelevance of the timescale of free fall $\tau_{c}=\sqrt{H_{0} / g}$. Surprisingly, we showed that despite fundamentally different dynamics, macroscopic quantities of our avalanches of nonconvex particles such as the runout distance and the final height of the pile agree reasonably well with results from the literature obtained with spherical particles. We also showed that our numerical simulations reproduce every flow behavior observed experimentally and we obtained excellent quantitative agreement between our simulations and our experiments. In particular, we emphasized that the use of a contact model with memory is critical as soon as there is a subsystem in a pseudostatic state, in our case, the bottom part of the granular pile. Futures studies should investigate avalanches of very large systems of entangled particles (of the order of $10^{6}$ particles) in order to reduce the uncertainty on the measured runout distance and final height.

Using our validated simulations, we investigated the contact force magnitude distribution and the fabric anisotropy in the granular assemblies before opening the gate. These data cannot be measured experimentally and are accessible via numerical simulations only. Our analysis of the average PDFs of the scaled contact force magnitude revealed that the distribution of forces in the case of cross-shaped particles is considerably broader than in the case of spheres and that the network of strong forces does not form a backbone in the case of cross-shaped particles. Our attempt to discriminate the top-driven collapse from the buckling collapse as well as the intermittent-stable regime from the intermittent-collapsed regime by means of PDFs of contact force magnitude and fabric anisotropy was not conclusive, suggesting that these collapse behaviors are governed by other properties of the granular assembly that at this stage we could not identify. Pursuing our investigation of the stability of granular assemblies in the intermittent regime, we found that quantities such as the average coordination number, the average number of contact points with the walls and with the gate, and the Janssen effect on the walls do not allow one to predict if an intermittent structure is about to collapse. This suggests that whether the structure collapses or remains stable is governed by mesoscale properties of the force network, such as local clustering. Future studies could investigate the mesoscale structures of granular networks of nonspherical particles and their implications on the dynamics of the system, as well as attempt to define an entanglement number as in [9] and investigate its relevance in describing the various observed behaviors. We finally discussed the choice of model parameters of our numerical simulations. We found that previously developed procedures used to set the tangential model parameters of spherical and ellipsoidal particles did not lead to physical behaviors when applied to our systems of angular nonconvex particles, resulting in a significant amount of time and resources spent to set the model parameters from trial and error. We emphasize the need for a reliable, physically based routine or calibration guidelines to set model parameters in a reproducible way, not only for spherical particles but also for particles of arbitrary shape.

\section{ACKNOWLEDGMENTS}

We greatly appreciate the financial support of the Natural Sciences and Engineering Research Council of Canada (NSERC) via A.W.'s Discovery Grant No. RGPIN-2016-06572. This research was enabled by support provided by Compute Canada through A.W.'s 2020 Computing Resources for Research Groups allocation qpf-764-ac. We also thank the anonymous reviewers for their comments and suggestions that have helped us to sharpen up the physical analysis presented in this paper.

\section{APPENDIX A: CONTACT MODEL IN ROTATION}

In the present study, the considered cross-shaped particles cannot roll and as a result our contact model does not include a friction torque. However, we also conduct simulations with systems of 
spherical particles, as shown in Fig. 12(a) for comparison purposes and in Appendix B for the sake of validation. Therefore, we present below the rotational contact model with memory together with the procedure adopted to set the rotational contact model parameters.

\section{Contact model formulation}

The friction torque $\boldsymbol{M}_{r}$ is modeled in a similar fashion as the tangential force and is a threedimensional adaptation of the two-dimensional work of Ai et al. [56],

$$
M_{r}=M_{k}+M_{d}
$$

with $\boldsymbol{M}_{\boldsymbol{k}}$ and $\boldsymbol{M}_{\boldsymbol{d}}$ defined incrementally as

$$
\begin{aligned}
& \boldsymbol{M}_{\boldsymbol{k}}^{t+\Delta t}= \begin{cases}q_{\mathrm{rot}} \boldsymbol{M}_{\boldsymbol{k}}^{t} q_{\mathrm{rot}}^{-1}-k_{r} \boldsymbol{\Delta} \boldsymbol{\theta} & \text { if }\left\|\boldsymbol{M}_{\boldsymbol{k}}^{t+\Delta t}\right\| \leqslant M_{\max } \\
M_{\max } \boldsymbol{u}_{r, \text { cumul }} & \text { otherwise, }\end{cases}
\end{aligned}
$$

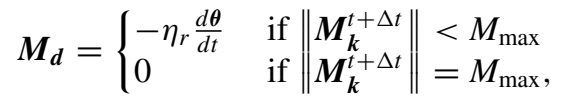

with

$$
\boldsymbol{u}_{r, \mathrm{cumul}}=\frac{\boldsymbol{M}_{\boldsymbol{k}}^{t}-k_{r} \boldsymbol{\Delta} \boldsymbol{\theta}}{\left\|\boldsymbol{M}_{\boldsymbol{k}}^{t}-k_{r} \boldsymbol{\Delta} \boldsymbol{\theta}\right\|}
$$

and

$$
M_{\max }=\mu_{r} R_{i j}\left\|\boldsymbol{F}_{n}\right\| .
$$

In the above equations, $k_{r}$ and $\eta_{r}$ are the rolling stiffness coefficient and the rolling damping coefficient, respectively, $\boldsymbol{\Delta} \boldsymbol{\theta}=\Delta t d \boldsymbol{\theta} / d t$ is the rotation increment, $\mu_{r}$ is a Coulomb-like static rolling friction coefficient, $M_{\max }$ is a saturation torque, $\boldsymbol{u}_{r, \text { cumul }}$ is the cumulative rolling direction unit vector, and $R_{i j}=R_{i} R_{j} /\left(R_{i}+R_{j}\right)$ is the reduced radius. Note that in accordance with Ai et al. [56], the saturation condition in Eqs. (A2) and (A3) only takes into account the springlike component $\boldsymbol{M}_{\boldsymbol{k}}$ of the total torque.

\section{Contact parameters in rotation}

In rotation, we set the contact parameters based on the approach of Jiang et al. [57], as it is developed from a credible micromechanical model. Since the contact between two rigid bodies never occurs over a point but rather over a surface, Jiang et al. [57] considered an infinite distribution of spring dashpots over this surface and derived a relation between the contact parameters in translation and in rotation. The friction torque between two spheres of identical radius $R$ is expressed as

$$
\boldsymbol{M}_{r}=\frac{\left(\delta_{r} R\right)^{2}\left(k_{n} \theta+\eta_{n} \dot{\theta}\right)}{12} \boldsymbol{u}_{r, \mathrm{cumul}}
$$

and

$$
\left\|\boldsymbol{M}_{r}\right\| \leqslant M_{\max }=\frac{1}{6} \delta R\left\|\boldsymbol{F}_{n}\right\|,
$$

with $\theta$ the relative rotation between the two rigid bodies and $\delta_{r}$ a quantity related to the surface area of contact, the so-called shape parameter, to be calibrated with experiments. Both rolling friction models from Ai et al. [56] and Jiang et al. [57] introduced a saturation torque $M_{\max }$ given by Eqs. (A5) and (A7), allowing a relationship between the shape parameter $\delta_{r}$ and the static rolling friction coefficient $\mu_{r}$ :

$$
\delta_{r}=6 \mu_{r}
$$



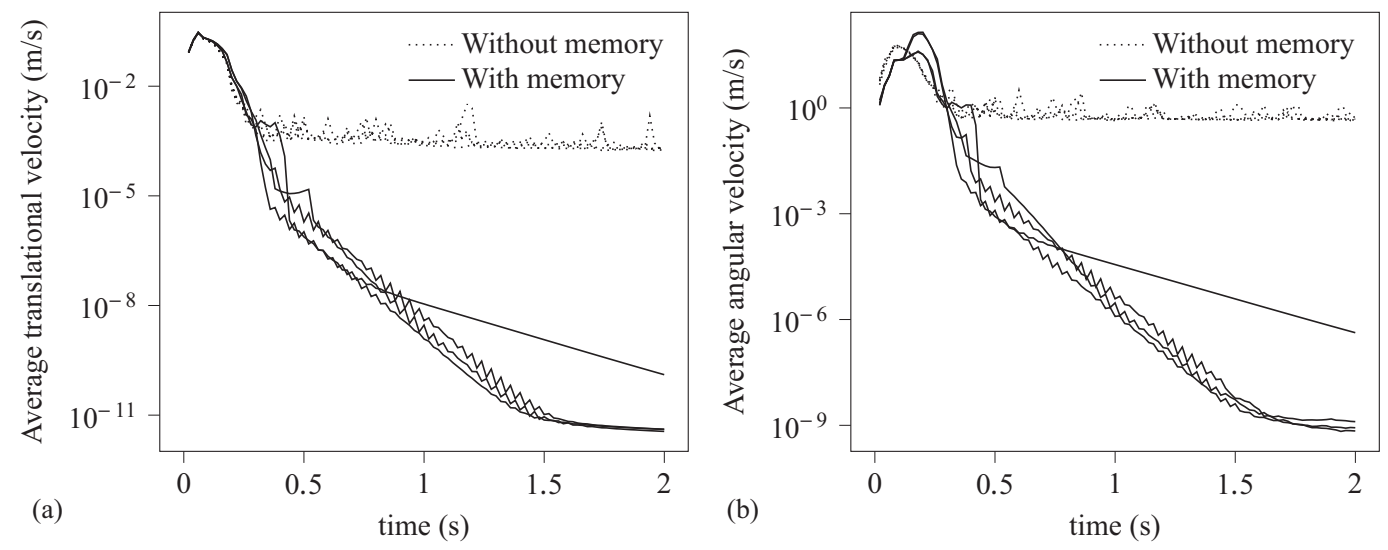

FIG. 22. Time evolution of the average (a) translational velocity and (b) angular velocity for a system of 750 spheres settling in a box. The dotted line represents no memory term in the contact model and the solid line represents a memory term in the contact model.

From Eqs. (A6) and (A8), the rolling contact parameters can be related to the normal contact parameters through the static rolling friction coefficient:

$$
\begin{aligned}
& k_{r}=3 k_{n} \mu_{r}^{2} R^{2}, \\
& \eta_{r}=3 \eta_{n} \mu_{r}^{2} R^{2} .
\end{aligned}
$$

Note that the parameter $\mu_{r}$ still needs to be calibrated with experiments, but its physical meaning is clearer than the physical meaning of the shape parameter $\delta_{r}$,

$$
\mu_{r}=\tan \beta \text {, }
$$

where $\beta$ is the maximum slope angle at which the rolling friction torque balances the torque due to gravity [56].

\section{APPENDIX B: VALIDATION OF THE NUMERICAL METHOD}

The code GRAINS3D used in this study has successfully reproduced numerous experimental results in the past in the case of a contact model without memory $[16,17,28,29,58]$. Here we only seek validation of the memory contact model presented in Secs. II B 1 and A. We perform this validation for systems of spherical particles that are the most common systems studied in the literature.

\section{Quantifying the residual velocity}

The main motivation to introduce the history terms $k_{t} \boldsymbol{\delta}_{t}$ and $k_{r} \boldsymbol{\Delta} \boldsymbol{\theta}$ from Eqs. (6) and (A2) is to allow the system to reach a static state. Without these terms, the translational and rotational velocities never reach values close to the machine epsilon. Figure 22 shows how this contact model with history dramatically improves the static state of a system of 750 spheres settling in a box. Each line corresponds to one simulation with random initial positions of the spheres. We can see that for some of the randomly generated initial conditions it may take longer to reach a static state, but the static state is eventually reached. For a simulation time sufficiently large, the residual velocity of the particles is orders of magnitude lower when the contact model includes the history terms: lower than $10^{-10} \mathrm{~m} / \mathrm{s}$ versus larger than $10^{-4} \mathrm{~m} / \mathrm{s}$ in translation and lower than $10^{-8} \mathrm{rad} / \mathrm{s}$ versus larger than $10^{-1} \mathrm{rad} / \mathrm{s}$ in rotation. These results show that the contact model with history is at least able to yield true static states where the residual velocity of the particles is as close as possible to the 
TABLE II. Particle properties and contact model parameters of the Hopper discharge simulations.

\begin{tabular}{lccc}
\hline \hline Name & Symbol & Value & Unit \\
\hline particle density & $\rho$ & $2.5 \times 10^{3}$ & $\mathrm{~kg} / \mathrm{m}^{3}$ \\
particle radius & $R$ & $10^{-3}$ & $\mathrm{~m}$ \\
normal stiffness (giving $\left.\delta_{\max }=0.005 \times R\right)$ & $k_{n}$ & $\approx 7.34 \times 10^{5}$ & $\mathrm{~N} / \mathrm{m}$ \\
normal restitution coefficient & $e_{n}$ & 0.45 & \\
tangential stiffness & $k_{t}$ & $\approx 2.26 \times 10^{5}$ & $\mathrm{~N} / \mathrm{m}$ \\
tangential damping factor & $\gamma_{t}$ & $\approx 4.0 \times 10^{4}$ & $\mathrm{~s}^{-1}$ \\
particle-particle static friction coefficient & $\mu_{\mathrm{c}, \text { part-part }}$ & $0.05-0.65$ & \\
particle-wall static friction coefficient & $\mu_{\mathrm{c} \text {,part-wall }}$ & 0.45 & \\
particle-particle rolling friction coefficient & $\mu_{\mathrm{r}, \text { part-part }}$ & 0.45 & \\
particle-wall rolling friction coefficient & $\mu_{\mathrm{r} \text {,part-wall }}$ & 0.1 & $\mathrm{~s}$ \\
time step & $\Delta t$ & $5.0 \times 10^{-7}$ & \\
\hline \hline
\end{tabular}

machine epsilon. In the next section, we validate this model further and show that it reproduces physical results for the classical test case of the hopper discharge.

\section{Hopper discharge}

For this second test case, we consider a hopper discharge performed several times with various translational static friction coefficients $\mu_{c}$. As $\mu_{c}$ increases, we measure the repose angle of the pile. The details of the simulation parameters are presented in Table II. The particle density $\rho$, particle radius $R$, and normal restitution coefficient $e_{n}$ are chosen to match those of Yan et al. [45], while the rest of the coefficients are determined using the procedure described in Sec. II C 2.

Our simulation results are shown in Fig. 23, together with the results from Yan et al. The agreement is satisfactory: Both curves follow the same increasing trend and show the same saturation behavior, with a repose angle of about $36^{\circ}$ for $\mu_{c}=0.5$. One can notice that for $\mu_{c}$ in the range $[0.05,0.20]$ the slope is steeper in our simulations than in the numerical study of Yan et al. This discrepancy may be explained by the use of a different contact model (Yan et al. used the

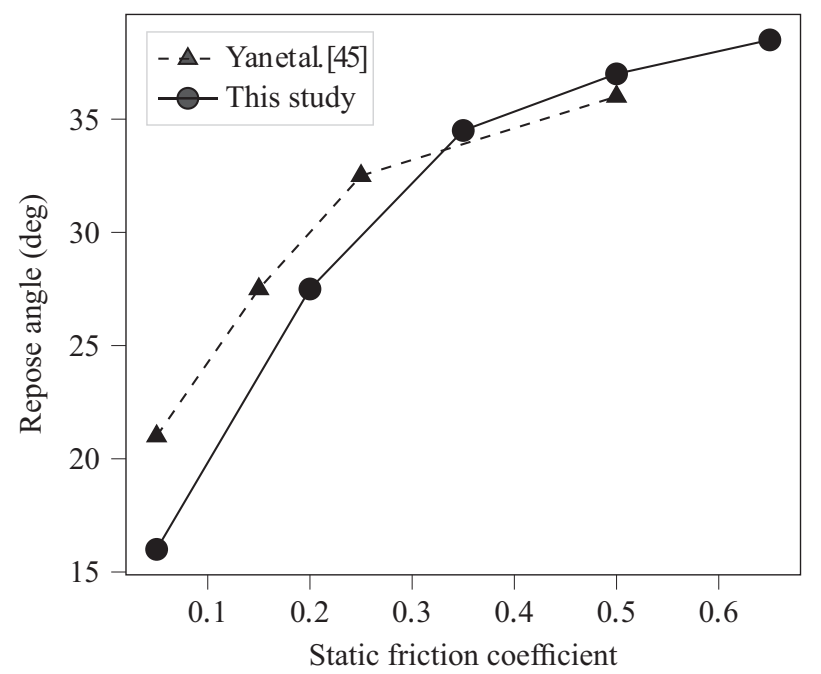

FIG. 23. Variation of the repose angle $\theta$ with the static friction coefficient $\mu_{c}$. The solid line shows results from the present study and the dashed line shows results adapted from Yan et al. [45]. 


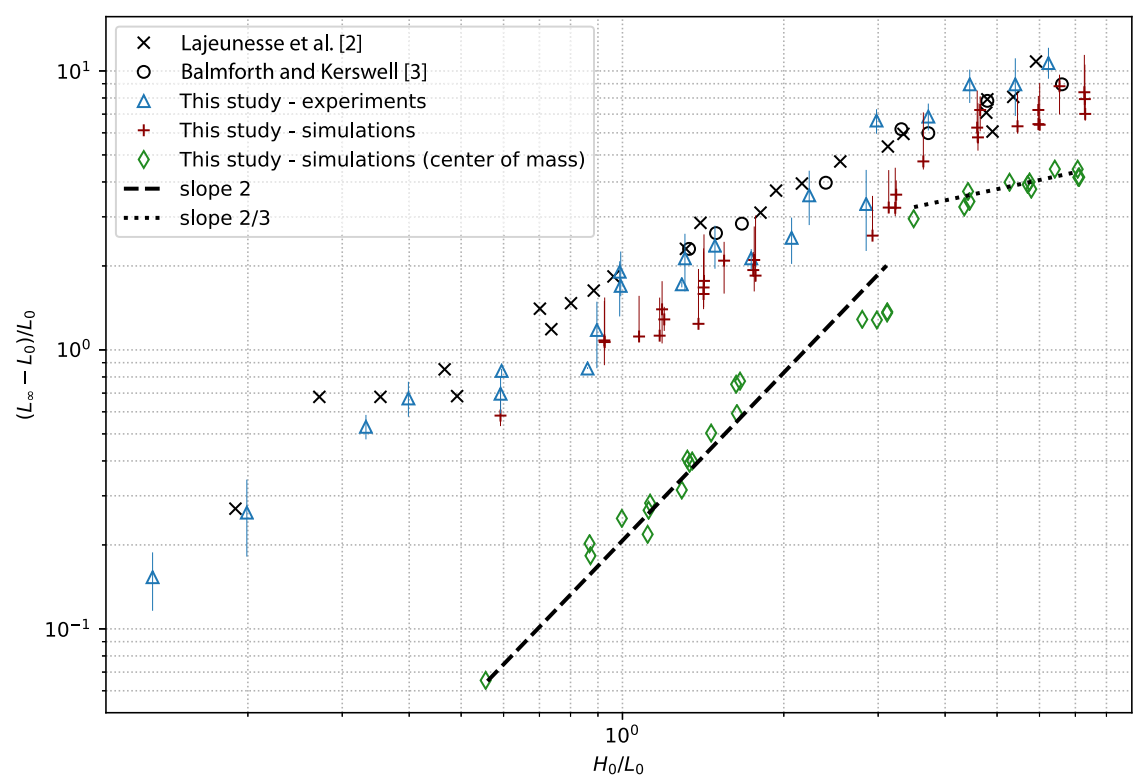

FIG. 24. Nondimensional final $x$ displacement of the center of mass of the granular assembly (diamonds) and nondimensional runout distance (all other symbols) as a function of the initial aspect ratio.

Hertz-Mindlin model) or by a slightly different setup, for instance, with regard to the opening of the gate.

\section{APPENDIX C: NONDIMENSIONAL DISPLACEMENT OF THE CENTER OF MASS OF THE WHOLE GRANULAR ASSEMBLY}

In a dam break setup, the measurement of the runout distance may be prone to uncertainties stemming from the dynamics of a few particles located at the front of the avalanche, in particular when the system is small. While we attempted to circumvent this limitation through a systematic image-processing routine and were conservative with the associated error bars (see Sec. IIIC), Degaetano et al. [59] instead reported the position of the center of mass of the granular assembly, a quantity that is far less noisy and less dependent on the dynamics of a few particles at the front of the avalanche. In fact, in their study of two-dimensional avalanches of bidisperse particles, Degaetano et al. obtained clearer trends when plotting the center of mass of the assembly rather than the runout distance. In this Appendix we investigate if reporting the position of the center of mass of our assemblies of monodisperse nonconvex particles rather than their runout distance yields a similar improvement of the interpretation of our simulation data.

Figure 24 shows the nondimensional final displacement of the center of mass as a function of the initial aspect ratio, as well as the nondimensional runout distance in our simulations, in our experiments, and in the experiments with spherical particles of Lajeunesse et al. [2] and with grit particles of Balmforth and Kerswell [3]. Focusing on the nondimensional displacement of the center of mass, we observe a very pronounced and clearly visible transition around $H_{0} / L_{0}=3$ where the slope suddenly changes from 2 to $2 / 3$. We show below how the numerical values of these slopes can be recovered by means of a simple model. While in Fig. 24 the slopes of the runout distance in our simulations and experiments seem constant and equal to 1, Lajeunesse et al. considered aspect ratios of up to 20 and revealed that indeed at $H_{0} / L_{0} \approx 3$ a slope transition from 1 to $2 / 3$ occurs. Figure 24 confirms that in our simulations of monodisperse nonconvex particles the displacement of the center of mass seems to be a more relevant quantity to identify the regime transition at 


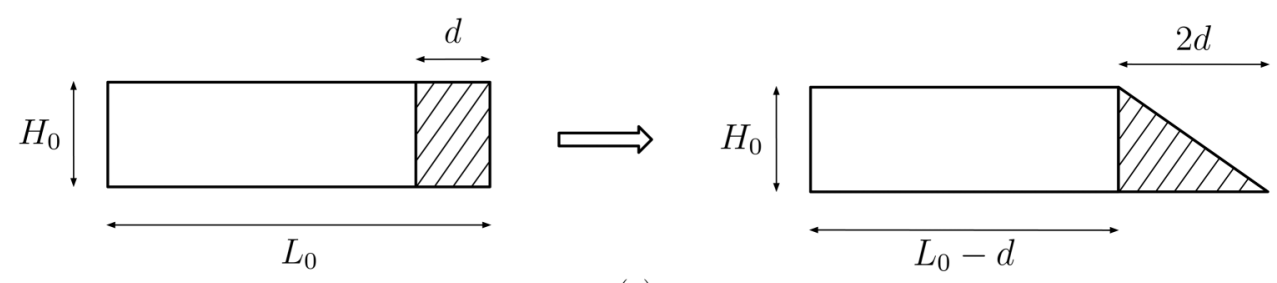

(a)
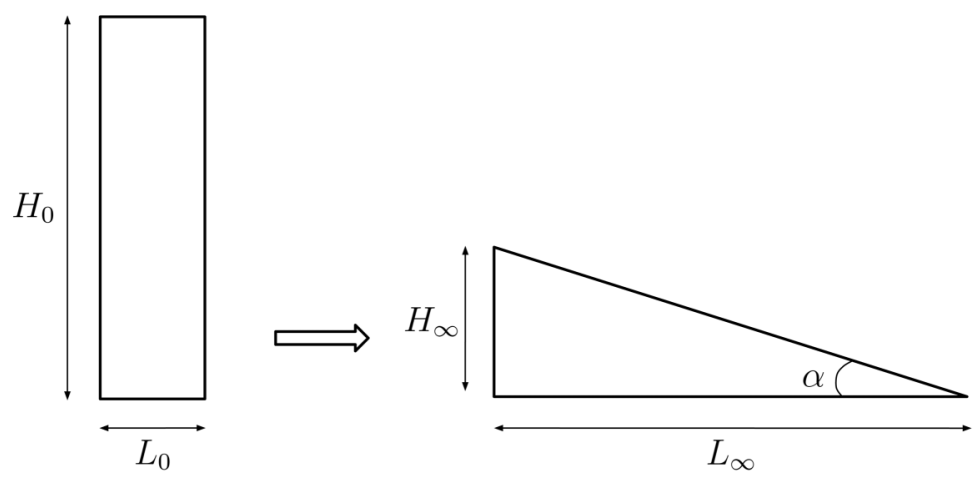

(b)

FIG. 25. Schematics of the avalanche profiles of the granular assembly for (a) low and (b) high aspect ratios, under the assumption that the slope of the avalanched profile is constant for a given aspect ratio.

$H_{0} / L_{0} \approx 3$. Therefore, focusing on the center of mass of the assembly improves the interpretation of the simulation data. Note that this observation is presumably true for any particle shape.

We now show with a simple model how the slopes in Fig. 24 can be recovered. For both regimes of small and large aspect ratios, we make the strong assumption that the slope of the avalanched region is constant, as formulated by Lajeunesse et al. [2] and roughly verified experimentally in Fig. 6. This assumption seems to not be valid in the case of buckling collapses (see Fig. 7), but since this regime occurs far less often that the top-driven collapse we can still consider the assumption of constant slope satisfied.

Case of small aspect ratios. In this case, the shape of the granular assembly before and after the collapse is represented in Fig. 25(a). We make the additional assumption that only the particles located at a distance less than $d$ away from the gate experience the avalanche and that $d$ is proportional to $H_{0}$ (most likely we have $d \approx H_{0}$, but this is not needed for the dimensional scaling). This assumption is again roughly justified by the results in our experiments and simulations. The nondimensional runout distance $\Delta L / L_{0}=\left(L_{\infty}-L_{0}\right) / L_{0}$ equals $d / L_{0}$ and is therefore proportional to $H_{0} / L_{0}$, hence the slope of 1 observed in Fig. 24. To estimate the nondimensional $x$ displacement of the center of mass, $\Delta x / x_{0}=\left(x_{\infty}-x_{0}\right) / x_{0}$, we need to first compute its final position $x_{\infty}$ with respect to its initial position $x_{0}=L_{0} / 2$. Simply adding the positions of the centers of mass of the plain white rectangle and the hatched triangle on the right-hand side of Fig. 25(a) and weighting them appropriately yields

$$
x_{\infty}=\left(\frac{L_{0}-d}{2}\right)\left(1-\frac{d}{L_{0}}\right)+\left(L_{0}+\frac{d}{3}\right)\left(\frac{d}{L_{0}}\right)=x_{0}\left(1+\frac{1}{3} \frac{d^{2}}{L_{0}^{2}}\right) \Rightarrow \frac{\Delta x}{x_{0}} \propto\left(\frac{H_{0}}{L_{0}}\right)^{2}
$$

which is in agreement with the slope of 2 observed in Fig. 24 for the center of mass for aspect ratios $H_{0} / L_{0} \lesssim 3$. 


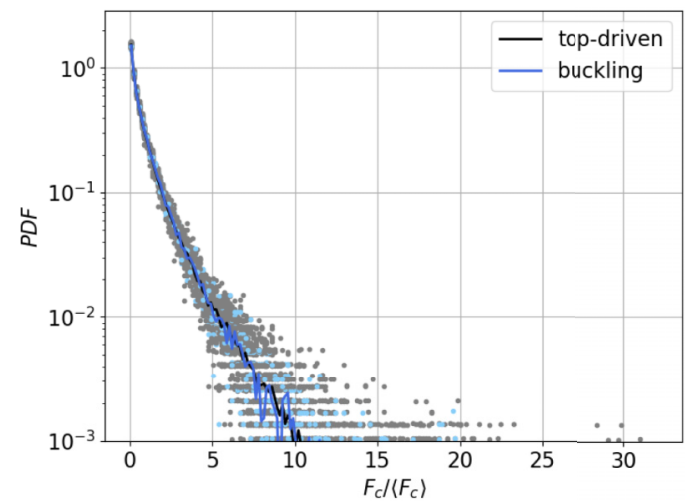

(a)

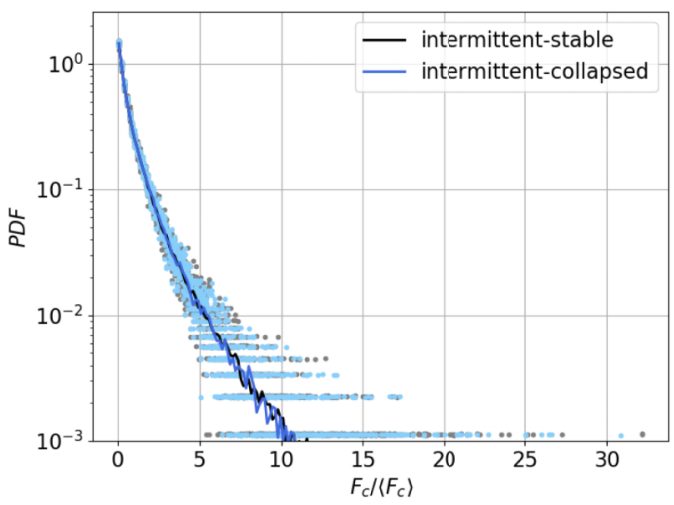

(c)

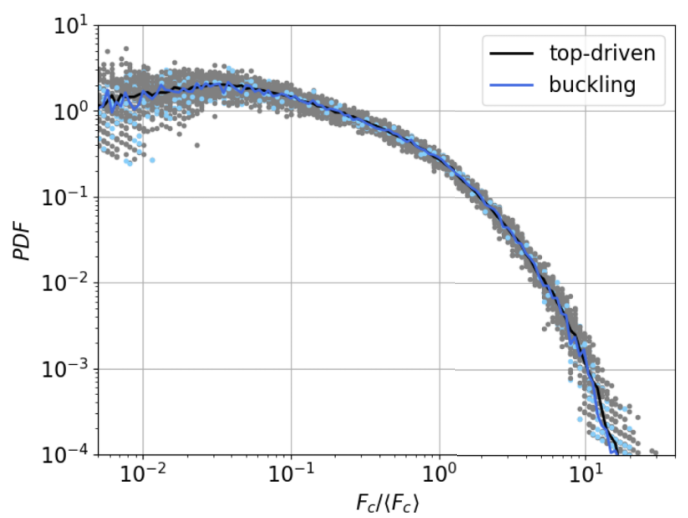

(b)

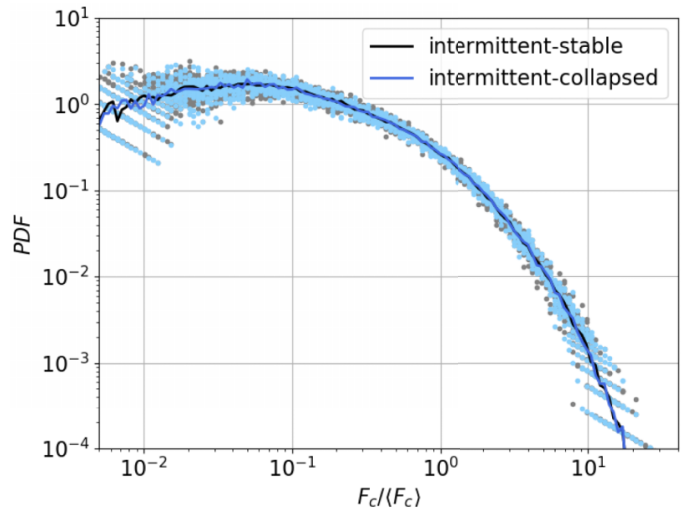

(d)

FIG. 26. Probability density functions of the contact forces $F_{c}$ just before the gate is opened, in (a) and (c) linear scale and (b) and (d) logarithmic scale, for the cases of (a) and (b) a top-driven regime (black) with a buckling regime (blue) and (c) and (d) an intermittent regime leading to stable (black) and collapsed (blue) behaviors.

Case of large aspect ratios. The case of high aspect ratios is schematically represented in Fig. 25(b), where $\alpha$ denotes the angle of the collapsed profile, which may vary with the aspect ratio $H_{0} / L_{0}$. Mass conservation yields $H_{0} L_{0}=H_{\infty} L_{\infty} / 2$, and since $H_{\infty}=\tan \alpha L_{\infty}$ we obtain

$$
\frac{\Delta L}{L_{0}}=\frac{L_{\infty}-L_{0}}{L_{0}}=\sqrt{\frac{2 H_{0}}{L_{0} \tan \alpha}} .
$$

Lajeunesse et al. have shown experimentally and by means of a force balance model [2] that the nondimensional runout distance $\Delta L / L_{0}$ scales as $\left(H_{0} / L_{0}\right)^{2 / 3}$ for large aspect ratios. This result implies that $\tan \alpha$ is proportional to $\left(H_{0} / L_{0}\right)^{-1 / 3}$. Focusing now on the center of mass, we have $x_{0}=L_{0} / 2$ and $x_{\infty}=L_{\infty} / 3$, and we use the mass conservation identity again to yield

$$
x_{\infty}=\frac{1}{3} \sqrt{\frac{2 H_{0} L_{0}}{\tan \alpha}} \Rightarrow \frac{\Delta x}{x_{0}}=\frac{x_{\infty}-x_{0}}{x_{0}}=\frac{1}{3} \sqrt{\frac{2 H_{0}}{L_{0} \tan \alpha}}-1 .
$$

Since tan $\alpha$ is proportional to $\left(H_{0} / L_{0}\right)^{-1 / 3}$ we recover the slope of $2 / 3$ that we observe in Fig. 24 for large aspect ratios. 
TABLE III. Average data for the intermittent cases (a) about to collapse and (b) remaining stable. The symbol $\sigma$ stands for the standard deviation of the data.

\begin{tabular}{lccc}
\hline \hline Name & Whole space & $\begin{array}{c}\text { Half space close } \\
\text { to the gate }\end{array}$ & $\begin{array}{c}\text { Quarter space close } \\
\text { to the top of the gate }\end{array}$ \\
\hline & $(\mathrm{a})$ & & \\
coordination number & $8.20(\sigma=0.07)$ & $8.20(\sigma=0.07)$ & $7.96(\sigma=0.11)$ \\
No. of contact points on the ground & $79.3(\sigma=4.0)$ & $39.3(\sigma=3.4)$ & 0 \\
No. of contact points on sidewalls & $169.2(\sigma=5.7)$ & $84.3(\sigma=3.6)$ & $33.0(\sigma=3.4)$ \\
No. of contact points on the gate & $72.8(\sigma=4.5)$ & $72.8(\sigma=4.5)$ & $26.1(\sigma=3.4)$ \\
& $8.21(\sigma=0.07)$ & $8.21(\sigma=0.09)$ & $8.02(\sigma=0.12)$ \\
coordination number & $81.2(\sigma=4.6)$ & $40.9(\sigma=3.1)$ & 0 \\
No. of contact points on the ground & $170.3(\sigma=5.3)$ & $85.4(\sigma=4.0)$ & $30.9(\sigma=2.5)$ \\
No. of contact points on sidewalls & $72.1(\sigma=3.1)$ & $72.1(\sigma=3.1)$ & $28.1(\sigma=2.7)$ \\
No. of contact points on the gate & & & \\
\hline \hline
\end{tabular}

\section{APPENDIX D: PROBABILITY DENSITY FUNCTIONS OF THE SCALED CONTACT FORCE MAGNITUDE IN VARIOUS REGIMES}

Figure 26 shows the average PDFs of $F_{c} /\left\langle F_{c}\right\rangle$ in the top-driven collapse and in the buckling collapse [Figs. 26(a) and 26(b)] and in the intermittent-stable regime and in the intermittent-collapsed regime [Figs. 26(c) and 26(d)]. Each solid line is an average of $N$ simulations. In Figs. 26(a) and 26(b), $N_{\text {top-driven }}=50$ and $N_{\text {buckling }}=6$, while in Figs. 26(c) and 26(d), $N_{\text {stable }}=26$ and $N_{\text {collapsed }}=$ 18. The low number of buckling cases is due to the scarcity of this regime in our simulations; the buckling collapse occurs in about $10 \%$ of the simulations located in the buckling region of Fig. 9. Overall, Fig. 26 shows that the average PDF of $F_{c} /\left\langle F_{c}\right\rangle$ in the buckling collapse and the average PDF of $F_{c} /\left\langle F_{c}\right\rangle$ in the top-driven collapse overlap very well; so do the average PDF of $F_{c} /\left\langle F_{c}\right\rangle$ in the intermittent-stable regime and the average PDF of $F_{c} /\left\langle F_{c}\right\rangle$ in the intermittent-collapsed regime. These results underline the fact that the distribution of force magnitude does not enable us to $a$ priori discriminate these regimes from one another and is not a relevant descriptor in predicting the behavior of the granular avalanche of our cross-shaped entangled particles.

\section{APPENDIX E: RAW DATA FOR THE ANALYSIS OF THE INTERMITTENT REGIME}

Table III shows the raw average contact data for a granular column in the intermittent regime. The forces on vertical walls are not included, since the Janssen effect is found to carry on average less than $0.3 \%$ of the total weight of the column.

[1] B. Andreotti, Y. Forterre, and O. Pouliquen, Granular Media: Between Fluid and Solid (Cambridge University Press, Cambridge, 2013).

[2] E. Lajeunesse, J. Monnier, and G. Homsy, Granular slumping on a horizontal surface, Phys. Fluids 17, 103302 (2005).

[3] N. Balmforth and R. Kerswell, Granular collapse in two dimensions, J. Fluid Mech. 538, 399 (2005).

[4] R. Kerswell, Dam break with Coulomb friction: A model for granular slumping? Phys. Fluids 17, 057101 (2005).

[5] P. W. Cleary and M. L. Sawley, DEM modelling of industrial granular flows: 3D case studies and the effect of particle shape on hopper discharge, Appl. Math. Model. 26, 89 (2002).

[6] P. W. Cleary, DEM prediction of industrial and geophysical particle flows, Particuology 8, 106 (2010). 
[7] M. D. Sinnott, P. W. Cleary, and R. D. Morrison, Is media shape important for grinding performance in stirred mills? Miner. Eng. 24, 138 (2011).

[8] S. Rémond, J. Gallias, and A. Mizrahi, Simulation of the packing of granular mixtures of non-convex particles and voids characterization, Granul. Matter 10, 157 (2008).

[9] N. Gravish, S. V. Franklin, D. L. Hu, and D. I. Goldman, Entangled Granular Media, Phys. Rev. Lett. 108, 208001 (2012).

[10] J. Landauer, M. Kuhn, D. S. Nasato, P. Foerst, and H. Briesen, Particle shape matters-Using 3D printed particles to investigate fundamental particle and packing properties, Powder Technol. 361, 711 (2020).

[11] S. Wang, D. Marmysh, and S. Ji, Construction of irregular particles with superquadric equation in DEM, Theor. Appl. Mech. Lett. 10, 68 (2020).

[12] L. Staron and E. J. Hinch, Study of the collapse of granular columns using two-dimensional discrete-grain simulation, J. Fluid Mech. 545, 1 (2005).

[13] G. Lube, H. E. Huppert, R. S. J. Sparks, and M. A. Hallworth, Axisymmetric collapses of granular columns, J. Fluid Mech. 508, 175 (2004).

[14] G. Lube, H. E. Huppert, R. S. J. Sparks, and A. Freundt, Collapses of two-dimensional granular columns, Phys. Rev. E 72, 041301 (2005).

[15] L. Lacaze, J. C. Phillips, and R. R. Kerswell, Planar collapse of a granular column: Experiments and discrete element simulations, Phys. Fluids 20, 063302 (2008).

[16] L. Girolami, V. Hergault, G. Vinay, and A. Wachs, A three-dimensional discrete-grain model for the simulation of dam-break rectangular collapses: Comparison between numerical results and experiments, Granul. Matter 14, 381 (2012).

[17] L. Girolami, A. Wachs, and G. Vinay, Unchannelized dam-break flows: Effects of the lateral spreading on the flow dynamics, Phys. Fluids 25, 043306 (2013).

[18] K.-W. Lim, K. Krabbenhoft, and J. E. Andrade, On the contact treatment of non-convex particles in the granular element method, Comput. Particle Mech. 1, 257 (2014).

[19] A. D. Rakotonirina and A. Wachs, Grains3D, a flexible DEM approach for particles of arbitrary convex shape - Part II: Parallel implementation and scalable performance, Powder Technol. 324, 18 (2018).

[20] E. Guendelman, R. Bridson, and R. Fedkiw, ACM Transactions on Graphics (ACM, New York, 2003), Vol. 22, pp. 871-878.

[21] L. Zhan, C. Peng, B. Zhang, and W. Wu, A surface mesh represented discrete element method (SMRDEM) for particles of arbitrary shape, Powder Technol. 377, 760 (2021).

[22] L. Rothenburg and R. J. Bathurst, Numerical simulation of idealized granular assemblies with plane elliptical particles, Comput. Geotech. 11, 315 (1991).

[23] X. Lin and T.-T. Ng, Contact detection algorithms for three-dimensional ellipsoids in discrete element modelling, Int. J. Numer. Anal. Methods Geomech. 19, 653 (1995).

[24] J. Williams and R. O'Connor, A linear complexity intersection algorithm for discrete element simulation of arbitrary geometries, Eng. Comput. 12, 185 (1995).

[25] G. Lu, J. Third, and C. Müller, Discrete element models for non-spherical particle systems: From theoretical developments to applications, Chem. Eng. Sci. 127, 425 (2015).

[26] G. Lu, J. Third, and C. Müller, Critical assessment of two approaches for evaluating contacts between super-quadric shaped particles in DEM simulations, Chem. Eng. Sci. 78, 226 (2012).

[27] A. Podlozhnyuk, S. Pirker, and C. Kloss, Efficient implementation of superquadric particles in discrete element method within an open-source framework, Comput. Part. Mech. 4, 101 (2017).

[28] A. Wachs, L. Girolami, G. Vinay, and G. Ferrer, Grains3D, a flexible DEM approach for particles of arbitrary convex shape - Part I: Numerical model and validations, Powder Technol. 224, 374 (2012).

[29] A. D. Rakotonirina, J.-Y. Delenne, F. Radjai, and A. Wachs, Grains3D, a flexible DEM approach for particles of arbitrary convex shape-Part III: Extension to non-convex particles modelled as glued convex particles, Comput. Part. Mech. 6, 55 (2019).

[30] L. J. H. Seelen, J. T. Padding, and J. A. M. Kuipers, A granular discrete element method for arbitrary convex particle shapes: Method and packing generation, Chem. Eng. Sci. 189, 84 (2018).

[31] F. Ludewig and N. Vandewalle, Strong interlocking of nonconvex particles in random packings, Phys. Rev. E 85, 051307 (2012). 
[32] V. Vivacqua, A. López, R. Hammond, and M. Ghadiri, DEM analysis of the effect of particle shape, cohesion and strain rate on powder rheometry, Powder Technol. 342, 653 (2019).

[33] N. Govender, D. N. Wilke, C.-Y. Wu, J. Khinast, P. Pizette, and W. Xu, Hopper flow of irregularly shaped particles (non-convex polyhedra): GPU-based DEM simulation and experimental validation, Chem. Eng. Sci. 188, 34 (2018).

[34] P. A. Cundall and O. D. L. Strack, A discrete numerical model for granular assemblies, Géotechnique 29, 47 (1979).

[35] H. Kruggel-Emden, M. Sturm, S. Wirtz, and V. Scherer, Selection of an appropriate time integration scheme for the discrete element method (DEM), Comput. Chem. Eng. 32, 2263 (2008).

[36] R. Kačianauskas, D. Markauskas, A. Džiugys, and R. Navakas, in Proceedings of the 19th International Conference on Computer Methods in Mechanics. Warsaw, 2011, edited by A. Borkowski, T. Lewiński, and G. Dzierżanowski (Warsaw University of Technology Press, Warsaw, 2011).

[37] P. A. Cundall, Formulation of a three-dimensional distinct element model-Part I. A scheme to detect and represent contacts in a system composed of many polyhedral blocks, Int. J. Rock Mech. Mining Sci. Geomech. Abstracts 25, 107 (1988).

[38] E. G. Nezami, Y. M. A. Hashash, D. Zhao, and J. Ghaboussi, A fast contact detection algorithm for 3-D discrete element method, Comput. Geotech. 31, 575 (2004).

[39] E. G. Gilbert, D. W. Johnson, and S. S. Keerthi, A fast procedure for computing the distance between complex objects in three-dimensional space, IEEE J. Robot. Autom. 4, 193 (1988).

[40] G. Van den Bergen, A fast and robust GJK implementation for collision detection of convex objects, J. Graphics, GPU, Game Tools 4, 7 (1999).

[41] A. Džiugys and B. Peters, An approach to simulate the motion of spherical and non-spherical fuel particles in combustion chambers, Granular Matter 3, 231 (2001).

[42] A. Wachs, Particle-scale computational approaches to model dry and saturated granular flows of nonBrownian, non-cohesive, and non-spherical rigid bodies, Acta Mech. 230, 1919 (2019).

[43] S. Luding, Introduction to discrete element methods: Basic of contact force models and how to perform the micro-macro transition to continuum theory, Eur. J. Env. Civil Eng. 12, 785 (2008).

[44] P. Costa, B. J. Boersma, J. Westerweel, and W.-P. Breugem, Collision model for fully resolved simulations of flows laden with finite-size particles, Phys. Rev. E 92, 053012 (2015).

[45] Z. Yan, S. K. Wilkinson, E. H. Stitt, and M. Marigo, Discrete element modelling (DEM) input parameters: understanding their impact on model predictions using statistical analysis, Comput. Part. Mech. 2, 283 (2015).

[46] L. Pournin, T. M. Liebling, and A. Mocellin, Molecular-dynamics force models for better control of energy dissipation in numerical simulations of dense granular media, Phys. Rev. E 65, 011302 (2001).

[47] See, https://youtu.be/yzlzn_XrkJA.

[48] See, https://youtu.be/DBmOVcR9PrU.

[49] F. Radjai, D. E. Wolf, M. Jean, and J.-J. Moreau, Bimodal Character of Stress Transmission in Granular Packings, Phys. Rev. Lett. 80, 61 (1998).

[50] B. Saint-Cyr, J.-Y. Delenne, C. Voivret, F. Radjai, and P. Sornay, Rheology of granular materials composed of nonconvex particles, Phys. Rev. E 84, 041302 (2011).

[51] N. Estrada, A. Taboada, and F. Radjai, Shear strength and force transmission in granular media with rolling resistance, Phys. Rev. E 78, 021301 (2008).

[52] L. Papadopoulos, M. A. Porter, K. E. Daniels, and D. S. Bassett, Network analysis of particles and grains, J. Complex Netw. 6, 485 (2018).

[53] T. Roessler, C. Richter, A. Katterfeld, and F. Will, Development of a standard calibration procedure for the DEM parameters of cohesionless bulk materials-Part I: Solving the problem of ambiguous parameter combinations, Powder Technol. 343, 803 (2019).

[54] A. Abramian, L. Staron, and P.-Y. Lagrée, The slumping of a cohesive granular column: Continuum and discrete modeling, J. Rheol. 64, 1227 (2020).

[55] A. Bougouin, L. Lacaze, and T. Bonometti, Collapse of a liquid-saturated granular column on a horizontal plane, Phys. Rev. Fluids 4, 124306 (2019). 
[56] J. Ai, J. Chen, J. Rotter, and J. Ooi, Assessment of rolling resistance models in discrete element simulations, Powder Technol. 206, 269 (2011).

[57] M. Jiang, H.-S. Yu, and D. Harris, A novel discrete model for granular material incorporating rolling resistance, Comput. Geotech. 32, 340 (2005).

[58] F. Dorai, C. Moura Teixeira, M. Rolland, E. Climent, M. Marcoux, and A. Wachs, Fully resolved simulations of the flow through a packed bed of cylinders: Effect of size distribution, Chem. Eng. Sci. 129, 180 (2015).

[59] M. Degaetano, L. Lacaze, and J. C. Phillips, The influence of localised size reorganisation on shortduration bidispersed granular flows, Eur. Phys. J. E 36, 36 (2013). 